\title{
Atherosclerosis is attenuated by acacetin via Sirt1- mediated activation of AMPK/Sirt3 signals in diabetic ApoE-/- mice
}

\section{Wei-Min Han}

Xiamen University Medical College

\section{Xu-Chang Chen}

Xiamen University Medical College

\section{Yan Wang}

Xiamen University

Gui-Rong Li ( $\square$ grli8@outlook.com )

Xiamen University Medical College https://orcid.org/0000-0002-9437-2280

Original investigation

Keywords: acacetin, diabetes, atherosclerosis, Sirt1, Sirt3, AMPK

Posted Date: May 29th, 2020

DOI: https://doi.org/10.21203/rs.3.rs-30047/v1

License: (c) (i) This work is licensed under a Creative Commons Attribution 4.0 International License. Read Full License 


\section{Abstract}

\section{Background}

The strategy of decreasing atherosclerotic cardiovascular disorder is imperative to reduce premature death and improve quality of life in patients with diabetes mellitus. The present study was designed to investigate whether the natural flavone acacetin could improve diabetes- accelerated atherosclerotic lesions.

\section{Methods}

Diabetic model was established in 7-week-old $\mathrm{ApoE}^{-/-}$mice by intraperitoneal injection of STZ (daily $50 \mathrm{mg} / \mathrm{kg}$ ) for 5 days. Animals of control, control with acacetin treatment, STZ-diabetes, STZ-diabetes with acacetin treatment received acacetin prodrug subcutaneously $(20 \mathrm{mg} / \mathrm{kg}$, b.i.d.) or equivolume saline for 12 weeks, and the elasticity of carotid artery and the ability of vascular wall movement were determined with ultrasound and magnetic resonance imaging. Human umbilical vein endothelial cells (HUVECs) were cultured with medium containing $5.5 \mathrm{mM}$ or $33 \mathrm{mM}$ glucose and treated with acacetin or vehicle. Changes of related aortic lesions and signaling molecules were determined by biochemical and molecular approaches in animals and cultured HUVECs.

Results

It was found that acacetin significantly suppressed atherosclerotic lesions and neointima hyperplasia, improved the elasticity of carotid artery and the ability of vascular wall movement without reducing blood glucose level and reversed the impaired signaling molecules (i.e. SOD, Bcl2, PGC-1a, pAMPK, Sirt3 and Sirt1) in artery tissues in diabetic mice. In cultured HUVECs, high glucose-induced cell viability reduction, ROS over-production, decrease of anti-oxidation, increase of apoptosis, and impairment of mitochondrial function were countered by acacetin (0.3-3 $\mu \mathrm{M})$ in a concentration-dependent manner. Moreover, acacetin relies on Sirt1 activation by increasing NAMPT and NAD ${ }^{+}$followed by Sirt3, pAMPK and PGC-1a activation. Silencing Sirt1 abolished acacetin-induced activation of Sirt3, pAMPK, and PGC-1a.

\section{Conclusions}

These results indicate that Sirt1-mediated activation of pAMPK/Sirt3 signals is involved in protective effects of acacetin against atherosclerosis in diabetes by preserving mitochondrial function via reducing mitochondrial apoptosis and ROS production and enhancing its biogenesis, which suggests that acacetin may be a drug candidate for reducing atherosclerotic cardiovascular disorder in patients with diabetes.

\section{Background}

Decreasing atherosclerotic cardiovascular disorder is imperative to reduce premature death and improve quality of life in patients with diabetes mellitus, since atherosclerotic cardiovascular disease is the leading cause of increased mortality and an important cause of morbidity in patients with Type 2 
diabetes worldwide [1-3]. Sustained hyperglycemia (i.e. elevated blood glucose level) leads to macroand microvascular complications [4-6]. Experimental and clinical studies show that hyperglycemia accelerated the formation of atherosclerosis in diabetics [7-11]. Atherosclerosis results from diabetic macroangiopathy and characterized by endothelial injury and dysfunction followed by formation of new intra-plaque vessel due to excessive/abnormal neovasculogenesis and angiogenesis, increased vascular permeability of the capillary vessels and tissue edema followed by atherosclerotic plaque hemorrhage and plaque rupture [12].

Although the detailed mechanisms responsible for the accelerated formation of atherosclerosis lesion observed in diabetes are not fully understood [13-15], it is generally recognized that mitochondrial dysfunction and increased ROS production are involved in endothelial impairment and acceleration of atherosclerosis in diabetes [16-18]. The therapeutic strategies to treat diabetic atherosclerosis include better approaches to prevent, inhibit or reverse diabetic cardiovascular complications. Previous studies have demonstrated that endothelial protection is important against vascular disorders in diabetes mellitus [19-21]. Enhancing mitochondrial biogenesis and reducing mitochondrial ROS have emerged as crucial therapeutic approaches to ameliorate diabetic atherosclerosis injury [22].

We have previously found that the natural flavone acacetin (5,7-dihydroxy-4'-methoxyflavone), in addition to its atrial-selective anti-atrial fibrillation property $[23,24]$, is cardioprotective against ischemia/reperfusion or hypoxia/reoxygenation injury by its anti-oxidation, anti-inflammation, and antiapoptosis properties $[25,26]$. The present study was designed to investigate whether acacetin is protective against vascular injury in diabetic $\mathrm{ApoE}^{-/-}$mice induced by streptozotocin (STZ) and in cultured human umbilical vein endothelial cells (HUVECs) exposed to high glucose conditions. Our results suggested that acacetin slowed the development of atherosclerosis in STZ-diabetic ApoE ${ }^{-/-}$mice and improved the injury of high glucose-cultured HUVECs by Sirt1-mediated activation of AMPK/Sirt3 signals.

\section{Materials And Methods}

\section{Animal experiments}

The animal experimental protocol was approved by the Animal Care and Ethics Committee of Xiamen University. Male ApoE ${ }^{-/-}$mice were obtained from Beijing Vital River Laboratory Animal Technology (Beijing, China) and raised in Laboratory Animal Center of Xiamen University. The age-matched animals were cared following the Guide for Care and Use of Laboratory Animals published by National Institutes of Health (NIH Publication No. 85-23, revised 1996) United States. Diabetic model was established in 7week-old ApoE ${ }^{-/-}$mice by intraperitoneal injection of STZ (daily $50 \mathrm{mg} / \mathrm{kg}$, Sigma-Aldrich, MO, United States) or vehicle citric acid (control) for 5 consecutive days. Random blood glucose was determined 2 weeks after streptozotocin injection with the Accu-Chek Performa glucometer (Roche, United States), and only animals with blood glucose $>16.7 \mathrm{mM}$ were classified as diabetic. Experiments were assigned as control, control with acacetin treatment, STZ-diabetes, STZ-diabetes with treatment. Animals with acacetin treatment received acacetin prodrug subcutaneously at $20 \mathrm{mg} / \mathrm{kg}$ twice daily (the prodrug can 
be metabolized into acacetin) [24, 25], and other animals received subcutaneous equivolume vehicle ( $0.9 \%$ saline). All animals were maintained at room temperature $\left(23 \pm 2^{\circ} \mathrm{C}\right)$ with a $12 \mathrm{~h}$ light/dark cycle and free access to basic diet and water for additional 12 weeks. Bodyweight and blood glucose were measured every four-week. When the animals were sacrificed at end of experiments, the blood was collected in a centrifuged tube with $25 \mathrm{I}$ of heparin. After centrifugation, plasma was collected to analyze the amount of cholesterol and triglycerides and high-density lipoprotein, low-density lipoprotein, lipoprotein A, lipoprotein B, $\beta$-OHB with Infinity reagent (Thermo Fisher Scientific, Waltham, MA, United States). Aortas were isolated for immunohistochemistry, immunofluorescence, and western blot analyses.

\section{High-frequency ultrasound imaging.}

Vascular lesions were measured non-invasively under anesthetization with a High-frequency Ultrasound Imaging System (Vevo 2100 system with a MicroScan MS550 40-MHz transducer, Visualsonics, Toronto, Canada) as described previously [27] and in Supplemental Methods.

\section{Magnetic resonance imaging (MRI)}

The MRI was performed under anesthetization with a 9.4T Bruker Micro MRI (Pharma Scan, Ettlingen, Germany) to determine diameter of carotid artery as described in Supplemental Methods.

\section{Atherosclerotic assay}

Aortic root lesion and enface lesion areas of whole aorta were fixed with $4 \%$ paraformaldehyde and stained with Oil Red $O$ as described previously [28]. Aortic root sections ( $10 \mu \mathrm{m}$ thickness) of $4 \%$ paraformaldehyde-fixed, OCT-embedded frozen hearts were cut from the aortic valve leaflets at 150-200 $\mu \mathrm{m}$ following the valve leaflet. Sections were concurrently stained with $0.5 \% \mathrm{w} / \mathrm{v}$ Oil Red $\mathrm{O}$ and hematoxylin and eosin (HE) to assess atherosclerotic lesions as described previously [29]. The images were captured with an Olympus BX40 microscope (20x magnification). All image quantifications were analyzed as described previously [30].

\section{Cell culture and viability assay}

Human umbilical vein endothelial cells (HUVECs) were obtained from ScienCell Research Laboratories (San Diego, CA, United States) and cultured in plates pre-coated with $0.2 \%$ gelatin in endothelial cell medium (ECM) supplemented with $5 \%$ fetal bovine serum, $1 \%$ penicillin/streptomycin with $1 \%$ endothelial cell growth supplement (ScienCell) at $37^{\circ} \mathrm{C}, 5 \% \mathrm{CO}_{2}$. For viability assay, cells were seeded in 96-well plates and grew to $70-80 \%$ confluence, then exposed to normal $(5.5 \mathrm{mM})$ or high $(33 \mathrm{mM})$ glucose (Sigma-Aldrich) medium for 5 days in the absence or presence of acacetin (0.3-3 mM). Cell viability was determined by 3-(4,5-dimethyl-2-thiazolyl)-2,5-diphenyl-2-H-tetrazolium bromide (MTT) (Solarbio Technology, Beijing, China) assay as described previously [26]. Briefly, the cells were incubated with 0.5 $\mathrm{mg} / \mathrm{mL}$ MTT for $4 \mathrm{~h}$, and re-suspended in $150 \mathrm{~mL}$ of DMSO. Absorbance was measured at $575 \mathrm{~nm}$ using 
an Infinite M200 Pro Nanoquant (TECAN, Switzerland). The cells treated with DMSO were considered to be $100 \%$ viable.

\section{Immunofluorescence analysis}

Immunofluorescence analysis was used to identify Sirt3 expression levels on aortic root sections and cultured HUVECs with different treatment. Briefly, aortic root sections were stained with anti-Sirt3 and anti-CD31 antibody (Abcam, Cambridge, MA, United States). Following an overnight incubation with primary antibodies, aortic root sections or cells were washed three time with PBS, followed by a 1-h incubation with Alexa Fluor-conjugated secondary antibodies (Alexa Fluor ${ }^{\circledR}$ 488, A-11001 or A-11034; Alexa Fluor ${ }^{\circledR}$ 568, A-11004 or A-11011; Alexa Fluor ${ }^{\circledR}$ 633, A-21052 or A-21071) (Thermo Fisher Scientific, Waltham, MA, United States) at room temperature, then mounted on DAPI-containing mounting media (Solarbio Technology, Beijing, China).

HUVECs were plated on confocal culture dish and cultured, thereafter labelled with MitoTracker ${ }^{\circledR}$ Red CMXRos (1:5000) incubated at $37^{\circ} \mathrm{C}$ for 20 min, then washed with PBS three times and fixed in $4 \%$ paraformaldehyde (Sigma-Aldrich, P6148) for $10 \mathrm{~min}$. After permeabilization with $0.5 \%$ Triton X-100 (Sigma-Aldrich, X100) for 10 min, the cells were blocked with 10\% goat serum (Solarbio Technology, Beijing, China) at $37^{\circ} \mathrm{C}$ for $1 \mathrm{~h}$, then incubated with anti-Sirt3 antibody (Solarbio Technology, Beijing, China) at $4^{\circ} \mathrm{C}$ overnight. Images were captured with a laser scanning confocal microscope (Olympus FV3000, Xiamen University, Fujian, China).

\section{Flow cytometry analysis}

Flow cytometry analysis was employed to assay the viability, apoptosis, ROS production and mitochondrial transmembrane potential in HUVECs using a flow cytometer (Beckman Coulter, United States) as described in Supplemental Methods.

\section{Mitochondrial oxidative stress and functional evaluation}

The mitochondrial oxidative stress proteins (i.e. SOD activity and MDA content) were measured using commercially available kits (Jiancheng Institute of Bioengineering, Nanjing, China) as described previously [31]. The intracellular ATP level was determined using ATP Bioluminescence Assay Kit (Beyotime Technology, Shanghai, China).

\section{$\mathrm{NAD}^{+} / \mathrm{NADH}$ determination}

The harvested cells were rinsed with PBS twice and centrifuged at $4 \times 1000 \mathrm{rpm}$ for $10 \mathrm{~min}$, and $\mathrm{NAD}^{+}$and NADH levels were quantified using an EnzyChrom ${ }^{\mathrm{TM}} \mathrm{NAD}{ }^{+} / \mathrm{NADH}$ assay kit (Bioassay Systems, Hayward, CA, United States) following the manufacturer's instruction.

\section{Small interfering RNA (siRNA).}


The siRNA technique was used to silence specific genes in HUVECs. The cells with $40 \%-50 \%$ confluence were transfected with specific siRNA duplexes (Santa Cruz Biotechnology, CA, United States) using Lipofectamine RNAiMAX Reagent (Thermo Fisher Scientific, Waltham, MA, United States) following the manufacturer's instruction. After $48 \mathrm{~h}$ transfection of control siRNA (sc-37007), Sirt1 siRNA (sc-40986), or Sirt3 siRNA (sc-61555) (Santa Cruz Biotechnology, Dallas, TX, United States), and then incubated with $5.5 \mathrm{mM}$ or $33 \mathrm{mM}$ glucose culture medium in the absence or presence of $3 \mathrm{mM}$ acacetin for 5 days. The cells transfected with siRNA were collected for western blot analysis.

\section{Western blot analysis}

Western blot analysis was employed to determine the expression of specific proteins in aortic tissues and cultured HUVECs. Proteins of aortic tissue homogenate lysates or HUVECs lysates prepared in SDS lysis buffer were extracted with RIPA buffer supplemented with protease and phosphatase inhibitors on ice, and protein concentration was determined using the BCA protein assay kit (Solarbio, Beijing, China) as described previously $[25,26]$. The proteins of HUVEC mitochondria were isolated using the Mitochondria Isolation Kit for Cultured Cells (Thermo Fisher Scientific, Waltham, MA, United States) following the manufacturer's instruction. SDS-PAGE and transferring PVDF membranes (Bio-Rad, Hercules, CA, United States) were applied to the separation of proteins samples. The membranes were blocked and incubated with primary antibodies (1:1000) overnight at $4^{\circ} \mathrm{C}$ overnight. After washout, membranes were incubated with secondary antibody (1:10000) for $1 \mathrm{~h}$ at room temperature. Blots were visualized with $\mathrm{ECL}^{\mathrm{TM}}$ reagents (Advansta, Menlo Park, CA, United States), and the protein signals were captured with FluorChem E chemiluminescence detection system (ProteinSimple, San Jose, CA, United States). All cellular western blots were repeated at least five times, and the signal intensity of the immunoreactive bands was quantified using Image $\mathrm{J}$ software (NIH, Bethesda, MD, United States) and normalized to that of $\beta$-actin in each sample. The primary antibodies are followed: anti-pAMPKa (\#2535) and anti-AMPKa (\#2532) antibodies were from Cell Signaling (Danvers, MA, United States); anti- $\beta$-actin (sc-47778) was from Santa Cruz; anti-Sirt1 (ab32441) anti-Sirt3 (ab217319), anti-Bcl-2 (ab182858), anti-Bax (ab32503), anti-PGC-1a (ab54481), anti-SOD1 (ab16831), anti-SOD2 (ab16956) antibodies were from Abcam (Cambridge, United Kingdom).

\section{Statistical Analysis}

Statistical analyses were performed with GraphPad Prism 6.0 (GraphPad Software, Inc., San Diego, CA, United States). Results are presented as means \pm SEM. One-way ANOVA followed by Bonferroni post hoc test were used for comparison among groups. $\mathrm{P}$ values $<0.05$ were considered statistically significant.

\section{Results}

Acacetin ameliorated alterations of lipid profiles without reducing blood glucose in STZ-diabetic ApoE ${ }^{-/-}$ mice 
Lipid profiles changed significantly in STZ-diabetic $\mathrm{ApoE}^{-/-}$mice (Table 1). Triglyceride, total cholesterol, low-density lipoprotein, lipoprotein A, and lipoprotein B (in $\mathrm{mM}$ ) were increased from $1.95 \pm 0.13,14.22 \pm$ $0.82,0.09 \pm 0.03,14.10 \pm 1.40$, and $0.075 \pm 0.011$ in control respectively to $3.99 \pm 0.39,21.72 \pm 1.13,6.39$ $\pm 0.49,70.33 \pm 9.60$, and $0.154 \pm 0.013$ ( $n=8, P<0.01$ vs. control), while high-density lipoprotein was decreased from $0.73 \pm 0.05$ in control to $0.49 \pm 0.03$ in STZ-diabetic ApoE ${ }^{-/-}$mice. Acacetin treatment did not alter the basal lipid profiles in control animals (Table 1); however, it reversed increases in triglyceride, total cholesterol, low-density lipoprotein, lipoprotein A, and lipoprotein B to respectively $2.69 \pm 0.2,16.29 \pm$ $0.95,4.39 \pm 0.53,27.93 \pm 4.59$, and $0.078 \pm 0.013 \mathrm{mM}(\mathrm{n}=8-10, \mathrm{P}<0.01$ vs. STZ-diabetes), and reversed the decrease in high-density lipoprotein to $0.66 \pm 0.04 \mathrm{mM}$ in STZ-diabetes treated with acacetin $(n=8-$ $10, P<0.05$ vs. STZ-diabetes). However, the random blood glucose level was not significantly reduced in STZ-diabetes treated with acacetin $(23.1 \pm 2.4 \mathrm{mM}$ vs. $20.4 \pm 2.1 \mathrm{mM}$ of STZ-diabetes, $P=N S)$ and final bodyweight showed no differences between STZ-diabetic animals $(27.87 \pm 1.47 \mathrm{~g})$ and STZ-diabetic animals treated with acacetin $(25.91 \pm 1.24 \mathrm{~g})$ (Table 1$)$. These results suggest that acacetin ameliorates the alterations in lipid profiles without reducing blood glucose level in STZ-diabetic ApoE ${ }^{-/-}$mice.

Table 1

Changes in bodyweight and lipid profiles in $\mathrm{ApoE}^{-/-}$mice

\begin{tabular}{|c|c|c|c|c|}
\hline Group & Control $(n=8)$ & Acacetin $(n=8)$ & $S T Z(n=8)$ & STZ + acacetin $(n=8)$ \\
\hline Initial body weight (g) & $25.13 \pm 1.36$ & $25.01 \pm 1.47$ & $24.44 \pm 1.68$ & $24.69 \pm 1.81$ \\
\hline Final body weight (g) & $31.22 \pm 1.77$ & $29.35 \pm 1.82$ & $25.91 \pm 1.24$ & $27.87 \pm 1.47$ \\
\hline Weight change (g) & $6.09 \pm 1.56$ & $4.34 \pm 1.65$ & $1.47 \pm 1.46$ & $3.18 \pm 1.64$ \\
\hline $\mathrm{FBG}\left(\mathrm{mmol} \cdot \mathrm{L}^{-1}\right)$ & $6.2 \pm 1.8$ & $7.5 \pm 1.6$ & $18.4 \pm 1.8^{\star \star}$ & $17.8 \pm 1.7^{\star \star}$ \\
\hline $\mathrm{RBG}\left(\mathrm{mmol} \cdot \mathrm{L}^{-1}\right)$ & $7.5 \pm 1.4$ & $9.1 \pm 1.8$ & $23.1 \pm 2.4^{\star \star}$ & $20.4 \pm 2.1^{\star \star}$ \\
\hline$\beta \mathrm{OHB}\left(\mathrm{mmol} \cdot \mathrm{L}^{-1}\right)$ & $0.111 \pm 0.012$ & $0.100 \pm 0.005$ & $0.147 \pm 0.014^{*}$ & $0.106 \pm 0.013^{\#}$ \\
\hline $\mathrm{TG}\left(\mathrm{mmol} \cdot \mathrm{L}^{-1}\right)$ & $1.95 \pm 0.13$ & $2.18 \pm 0.15$ & $3.99 \pm 0.39^{\star \star}$ & $2.69 \pm 0.2^{\# \#}$ \\
\hline $\mathrm{TC}\left(\mathrm{mmol} \cdot \mathrm{L}^{-1}\right)$ & $14.22 \pm 0.82$ & $14.98 \pm 1.02$ & $21.72 \pm 1.13^{\star \star}$ & $16.29 \pm 0.95^{\#}$ \\
\hline $\mathrm{HDL}\left(\mathrm{mmol} \cdot \mathrm{L}^{-1}\right)$ & $0.73 \pm 0.05$ & $0.78 \pm 0.08$ & $0.49 \pm 0.03^{\star \star}$ & $0.66 \pm 0.04^{\#}$ \\
\hline $\mathrm{LDL}\left(\mathrm{mmol} \cdot \mathrm{L}^{-1}\right)$ & $0.09 \pm 0.03$ & $0.11 \pm 0.03$ & $6.39 \pm 0.49^{\star \star}$ & $4.39 \pm 0.53^{\star \star, \# \#}$ \\
\hline $\mathrm{LP}(\mathrm{A})\left(\mathrm{mmol} \cdot \mathrm{L}^{-1}\right)$ & $14.10 \pm 1.40$ & $18.25 \pm 2.10$ & $70.33 \pm 9.60^{\star \star}$ & $27.93 \pm 4.60^{\# \#}$ \\
\hline $\mathrm{LP}(\mathrm{B})\left(\mathrm{mmol} \cdot \mathrm{L}^{-1}\right)$ & $0.075 \pm 0.011$ & $0.076 \pm 0.010$ & $0.154 \pm 0.013$ & $0.078 \pm 0.013$ \\
\hline
\end{tabular}




\section{Acacetin attenuated carotid artery injury in STZ-diabetic ApoE ${ }^{-/-}$mice}

The carotid artery injury was determined with MRI (Fig. S1) in STZ-diabetic ApoE ${ }^{-/-}$mice. T1WI, T2WI and walls of left and right common carotid arteries in non-diabetic $\mathrm{ApoE}^{-/-}$mice showed even and slightly high signal without obvious abnormal signal, while in STZ-diabetic $\mathrm{ApoE}^{-/-}$mice, there were uneven high signal areas on T1WI and T2WI, and artery lumen was clearly narrowed (vascular stenosis). These symptoms were significantly improved in STZ-diabetic $\mathrm{ApoE}^{-/-}$mice treated with acacetin (Table 2). 
Table 2

Ultrasound and MRI parameters in $\mathrm{ApoE}^{-/-}$mice

$\begin{array}{lllll}\text { Group } & \text { Control }(n=8) & \begin{array}{l}\text { Acacetin }(n= \\ 8\end{array} & \operatorname{STZ}(n=8) & \begin{array}{l}\text { STZ + acacetin }(n= \\ 8\end{array}\end{array}$

Ultrasound parameters

$\begin{array}{lllll}\text { Intimal thickness }(\mathrm{mm}) & 0.0559 \pm & 0.0582 \pm & 0.0893 \pm & 0.0610 \pm 0.0054^{\# \#} \\ & 0.0048 & 0.0047 & 0.0045^{\star \star}\end{array} \quad$.

$\operatorname{LCCA} \operatorname{EDV}(\mathrm{mm} / \mathrm{s})$

$38.58 \pm 3.26$

$39.02 \pm 1.61$

$62.69 \pm 5.33^{\star \star}$

$50.35 \pm 2.61^{\#}$

LCCA PSV $(\mathrm{mm} / \mathrm{s})$

$278.22 \pm$

15.53

$272.94 \pm$

11.57

$408.67 \pm$

$17.89^{\star \star}$

$316.01 \pm 19.67^{\# \#}$

LCCA resistance index

$0.7577 \pm$

$0.7613 \pm$

0.0091

$0.8665 \pm$

$0.0077^{\star \star}$

$0.7965 \pm 0.0014^{\star *}, \# \#$

Distensibility (1/MPa)

$146.87 \pm$

$140.51 \pm$

11.34

$55.99 \pm 8.91^{* *}$

$110.16 \pm 11.10^{\# \#}$

Tangential Strain near

(\%)

$3.52 \pm 0.25 \quad 3.31 \pm 0.24$

$1.74 \pm 0.17^{\star *}$

$2.85 \pm 0.22^{\# \#}$

Tangential Strain far

(\%)

$0.93 \pm 0.09$

$0.95 \pm 0.04$

$0.26 \pm 0.04^{\star *}$

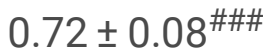

Radial strain (\%)

LCCV EDV $(\mathrm{mm} / \mathrm{s})$

RCCA PSV (mm/s)

RCCA resistance index

Distensibility (1/MPa)

Tangential Strain near (\%)

Tangential Strain far (\%)

Radial strain (\%)

$46.52 \pm 3.67$

$43.56 \pm 3.09$

$41.21 \pm 2.92$

$262.87 \pm$

10.75

$136.60 \pm 8.21$

$3.75 \pm 0.31$

$42.92 \pm 4.37$

$15.19 \pm 1.48^{\star \star}$

$33.18 \pm 2.92^{\# \#}$

$40.97 \pm 2.02$

$76.63 \pm 4.26^{\star \star}$

$48.10 \pm 4.67^{\# \#}$

$256.93 \pm$

$403.38 \pm$

$14.34^{\star *}$

$324.33 \pm 16.42^{\star *}, \# \#$

$\begin{array}{lll}0.7681 \pm & 0.7749 \pm & 0.8611 \pm \\ 0.0111 & 0.0077 & 0.0100^{\star \star}\end{array}$

$0.8084 \pm 0.0143^{*}, \# \#$

$137.28 \pm 9.29$

$71.64 \pm 7.24^{\star *}$

$97.92 \pm 9.22^{* *, \#}$

$3.66 \pm 0.28$

$1.87 \pm 0.15^{\star \star}$

$2.63 \pm 0.16^{\star \star}, \#$

MRI parameters

LCCA EDV,left common carotid artery end-diastolic velocity; LCCA PSV peak systolic velocity; RCCA EDV, right common carotid artery end-diastolic velocity; RCCA PSV, right common carotid artery peak systolic velocity. ${ }^{*} P<0.05,{ }^{* *} P<0.01$ vs. control; ${ }^{\#} P<0.05,{ }^{\# \#} P<0.01$ vs. STZ. 


\begin{tabular}{|c|c|c|c|c|}
\hline Group & Control $(n=8)$ & $\begin{array}{l}\text { Acacetin }(n= \\
\text { 8) }\end{array}$ & STZ $(n=8)$ & $\begin{array}{l}\text { STZ + acacetin }(n= \\
\text { 8) }\end{array}$ \\
\hline Diameter of LCCA (cm) & $0.475 \pm 0.013$ & $0.468 \pm 0.009$ & $0.340 \pm 0.019^{* \star}$ & $0.418 \pm 0.024^{\# \#}$ \\
\hline Diameter of RCCA $(\mathrm{cm})$ & $0.480 \pm 0.006$ & $0.472 \pm 0.011$ & $0.358 \pm 0.023^{\star \star}$ & $0.425 \pm 0.012^{\# \#}$ \\
\hline \multicolumn{5}{|c|}{$\begin{array}{l}\text { LCCA EDV,left common carotid artery end-diastolic velocity; LCCA PSV left common carotid artery } \\
\text { peak systolic velocity; RCCA EDV, right common carotid artery end-diastolic velocity; RCCA PSV, right } \\
\text { common carotid artery peak systolic velocity. }{ }^{\star} P<0.05,{ }^{\star \star} P<0.01 \text { vs. control; }{ }^{\#} P<0.05,{ }^{\# \#} P<0.01 \text { vs. } \\
\text { STZ. }\end{array}$} \\
\hline
\end{tabular}

Ultrasound was used for determining the wall thickness of aortic arch and blood flow velocity of left and right carotid arteries (Fig. S2) in $\mathrm{ApoE}^{-/-}$mice. The wall thickness of aortic arch and the artery distensibility measured by ultrasound biomicroscopy showed that the intimal thickness of aortic arch was increased and the carotid artery distensibility was decreased in STZ-diabetic mice, these effects were significantly countered in STZ-diabetic mice treated with acacetin (Table 2).

An earlier study revealed that carotid blood flow velocity was elevated as the artery stenosis became marked [32]. Here we also found that the diastolic and systolic blood flow velocities of left and right carotid arteries were significantly increased due to the artery stenosis, while the radial strain and tangential strain of left and right carotid arteries were reduced in the STZ-diabetic $\mathrm{ApoE}^{-/-}$mice. These alterations were countered in diabetic $\mathrm{ApoE}^{-/-}$mice treated with acacetin (Table 2). These results indicate that acacetin could improve the carotid artery stenosis thereby increasing the vascular radial strain and tangential strain and improving the movement ability of vascular walls.

The Oil Red $\mathrm{O}$ staining of enface aorta showed that atherosclerotic lesion was greater in STZ-diabetic $\mathrm{ApoE}^{-/-}$mice than nondiabetic $\mathrm{ApoE}^{-/-}$mice (control), and the lesion was reduced in STZ-diabetic ApoE ${ }^{-/}$ - mice treated with acacetin (Fig. 1A). The analysis of Oil Red $O$ stained area (Fig. 1B) exhibited that acacetin treatment significantly decreased the aorta lesion area from $11.0 \pm 1.12 \%(n=11, P<0.01)$ in non-treated STZ-diabetic $\mathrm{ApoE}^{-/-}$mice to $7.0 \pm 0.6 \%$.

The aortic root sections were stained with hematoxylin and eosin (Fig. 1C) or Oil Red O (Fig. 1E) to show the neointimal thickness and the lesion burden, in aortic roots of animals with different treatment. The increase in neointimal thickness (Fig. 1D) and lesion burden (Fig. 1F) were significantly reduced in STZ-

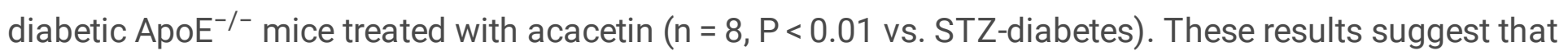
acacetin provides effective vascular protection against hyperglycemia-induced injury in STZ-diabetic $\mathrm{ApoE}^{-/-}$mice.

Acacetin reversed the downregulation of related protective molecule signals in aortic tissues of STZdiabetic ApoE ${ }^{-/-}$mice 
It is well documented that diabetic atherosclerosis is associated with downregulation of a series of signaling molecules involved in energy metabolism, anti-oxidation and anti-apoptosis, including Sirt1, AMPK, PGC-1a, SOD1, SOD2, Bcl-2, etc. [33, 34]. To investigate whether the vascular protection of acacetin is mediated by upregulating these signaling molecules, we determined the expression of Sirt1, PAMPK, PGC-1a, SOD1, SOD2, Bcl-2, and also Sirt3 in aortic tissues in non-diabetic mice, STZ-diabetic $\mathrm{ApoE}^{-/-}$mice, and STZ-diabetic ApoE ${ }^{-/-}$mice treated with acacetin (Fig. 2). As reported previously, the signaling molecules Sirt1, PGC-1a, SOD1, SOD2, and also Sirt3 were remarkably reduced (Fig. 2A \& 2B), pAMPK was decreased (Fig. 2D), the anti-apoptotic protein Bcl-2 was downregulated, and the proapoptotic Bax was increased (Fig. 2A \& 2C) in STZ-diabetic ApoE ${ }^{-/-}$mice $(n=8, P<0.01$ vs. control). The alterations in the expression of these molecules were reversed in STZ-diabetic $\mathrm{ApoE}^{-/-}$mice treated with acacetin (Fig. 2A-2D) ( $<<0.01$ vs. STZ-diabetes). These results suggest that the natural flavone acacetin confers vascular protection against diabetic atherosclerosis by reversing the downregulation of Sirt1, PGC-1a, SOD1, SOD2, Sirt3, pAMPK, and ratio of Bcl-2/Bax. Moreover, we first reported the reduced Sirt3 is involved in diabetic atherosclerosis. The immunohistochemical results showed that Sirt3 remarkably decreased on vascular wall, especially on endothelium stained with CD31, in STZ-diabetic ApoE ${ }^{-/-}$mice, which partially reversed in animals treated with acacetin (Fig. 2E).

\section{Acacetin prevents the viability reduction and increases in apoptosis and oxidative stress induced by high glucose exposure in HUVECs}

To investigate the potential molecular mechanism that vascular protection of acacetin against diabetic atherosclerosis, the related effects of acacetin were tested in HUVECs cultured with normal glucose concentration $(5.5 \mathrm{mM})$ medium or a high glucose concentration $(33 \mathrm{mM})$ medium. Acacetin $(0.3-3 \mu \mathrm{M})$ had no effect on viability in HUVECs cultured with normal glucose medium (Fig. 3A), while it reversed high glucose-induced viability reduction in a concentration-dependent manner (Fig. 3B). Flow cytometry analysis revealed that the viability reduction was related to high-glucose-induced increase in apoptosis (Fig. 3C), and acacetin significantly decreased the apoptosis (Fig. 3C and 3D). Results of western blot analysis showed that pro-apoptotic protein Bax was increased, while anti-apoptotic protein Bcl-2 was decreased in HUVECs cultured with $33 \mathrm{mM}$ glucose medium. Acacetin treatment reversed the Bax increase and enhanced the $\mathrm{Bcl}-2$ reduction, and increased the reduced $\mathrm{Bcl}-2 / \mathrm{Bax}$ ratio in a concentration dependent manner (Fig. 3E-3G). These results suggest that acacetin protects HUVECs against high glucose injury by inhibiting apoptosis.

It is generally believed that ROS overproduction is involved in endothelial apoptosis in diabetic cardiovascular complications. ROS production and the expression of antioxidant proteins SOD1 and SOD2 were therefore determined in HUVECs cultured with $33 \mathrm{mM}$ glucose medium (Fig. 4). High glucose culture induced an increase of ROS production in HUVECs,acacetin $(3 \mu \mathrm{M})$ significantly impeded the ROS production (Fig. 4A \& 4B). It reversed the increase in malondialdehyde (MDA) content (Fig. 4C) and reversed the reduction in SOD activity (Fig. 4D) in HUVECs cultured with $33 \mathrm{mM}$ medium in a concentration-dependent manner. Moreover, high glucose-induced reductions of SOD1 and SOD2 proteins were also reversed in HUVECs treated with acacetin (Fig. 4E \& 4F), 
To investigate the potential protection of acacetin against high glucose-induced endothelial mitochondrial injury, we determined mitochondrial transmembrane potential, ATP production and apoptosis-related proteins Bax and Bcl-2 in HUVECs (Fig. 5). The HUVECs cultured with $33 \mathrm{mM}$ glucose medium showed significant decrease of mitochondrial transmembrane potential and ATP production, and the reduction of mitochondrial transmembrane potential and ATP production was countered in cells treated with $3 \mu \mathrm{M}$ acacetin. Moreover, mitochondrial Bax (mitoBax) was increased, while mitochondrial Bcl-2 (mitoBcl-2) and Sirt3 (mitoSirt2) were reduced in HUVECs cultured with $33 \mathrm{mM}$ glucose medium ( $P$ $<0.01$ vs. $5.5 \mathrm{mM}$ glucose). The decreased ratio of mitoBcl-2/mitoBax and mitoSirt3 were counted ( $\mathrm{P}<$ 0.01 vs. $33 \mathrm{mM}$ glucose alone) in cells treated with $3 \mu \mathrm{M}$ acacetin (Fig. 5D-5G). Immunocytochemistry analysis also revealed the high glucose-induced decrease of mitoSirt3 was reversed in cells treated with 3 $\mu \mathrm{M}$ acacetin (Fig. $5 \mathrm{H})$. These results indicate that vascular protection of acacetin against high glucose injury is resulted from preserving mitochondria function.

\section{Silencing Sirt3 abolishes the protective effects of acacetin against high glucose-induced injury in HUVECs}

To determine the potential role of Sirt3 in acacetin protection against high glucose-induced injury, siRNA molecules targeting Sirt3 were employed in HUVECs. Figure 6 illustrates the effects of acacetin on high glucose-induced apoptosis, ROS production, and ATP reduction in HUVECs cultured with $33 \mathrm{mM}$ glucose medium and transfected with control siRNA or Sirt3 siRNA in the absence or presence of $3 \mu \mathrm{M}$ acacetin. Acacetin significantly decreased high glucose-induced apoptosis, ROS production in cells transfected with control siRNA, but not in cells transfected with Sirt3 siRNA. Also, acacetin increased ATP content in cells transfected with control siRNA, but not in cells transfected with Sirt3 siRNA. These results indicate that Sirt3 plays an important role in vascular protection against high glucose-induced injury, and also mediates mitochondrial ATP production.

Western blot analysis revealed that acacetin not only reversed the high glucose-induced decrease of Sirt3 in a concentration-dependent manner, but also the high glucose-induced downregulation of Sirt1, PGC-1a, and pAMPK (Fig. 7). These results indicate that vascular protective effect of acacetin is related to upregulating Sirt1, Sirt3, pAMPK, and PGC-1 a reduced by high glucose- culture in HUVECs or in STZdiabetic $\mathrm{ApoE}^{-/-}$mice.

\section{Protection of acacetin against hyperglycemia-induced injury is related to Sirt1-mediated activation of AMPK/Sirt3 signals in endothelial cells}

To further identify the molecule target of acacetin for the protection against high glucose- or hyperglycemia-induced vascular injury, siRNA molecules targeting to Sirt1 or Sirt3 and the AMPK inhibitor Compound C were utilized in HUVECs cultured with $33 \mathrm{mM}$ glucose medium to determine the effects of the siRNA molecules or AMPK inhibitor on acacetin-induced upregulation of Sirt1, Sirt3, pAMPK and PGC$1 \mathrm{a}($ Fig. 8A \& 8B). It is interesting to note that silencing Sirt1 abolished the acacetin-induced increase of Sirt1, Sirt3, pAMPK and PGC-1a, while silencing Sirt3 only inhibited the upregulation of Sirt3, but not the 
acacetin-induced increase of Sirt1, pAMPK and PGC-1a. The AMPK inhibitor Compound C decreased the expression of PAMPK, PGC-1 a, and Sirt3, and slightly reduced the Sirt3 increase by acacetin, whereas it fully abolished the increase of pAMPK and PGC-1a, but not Sirt1. These results indicate that protective effect of acacetin against hyperglycemia-induced vascular injury is related to Sirt1-mediated activation of AMPK, Sirt3 and PGC-1a signals in endothelial cells.

It is well recognized that sirtuins are nicotinamide adenine dinucleotide- $\left(\mathrm{NAD}^{+}-\right)$dependent deacetylases that controls metabolism, and biosynthesis of $\mathrm{NAD}^{+}$is mediated by nicotinamide phosphoribosyltransferase (NAMPT) [35]. The sirtuins activity is correlated to ratio of $\mathrm{NAD}^{+} / \mathrm{NADH}$ [36]. We therefore determined the potential effects of acacetin on the NAD ${ }^{+} / \mathrm{NADH}$ ratio and expression of Sirt1 and Sirt3 in the absence or presence of the NAMPT inhibitor GMX-1778 (CHS-828) [37]. The $\mathrm{NAD}^{+} / \mathrm{NADH}$ ratio was reduced in HUVECs cultured with $33 \mathrm{mM}$ glucose medium, and the reduction was

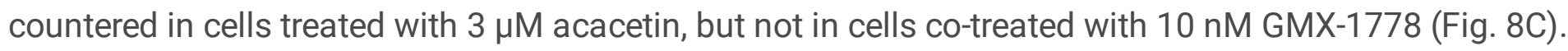
Moreover, GMX-1778 not only induced a further decrease of Sirt1 and Sirt3 proteins, but also prevented the acacetin induced increase of Sirt1 and Sirt3 proteins (Fig. 8D \& 8E) in HUVECs cultured with $33 \mathrm{mM}$ glucose medium. These results indicate that the vascular protection of acacetin against high glucose- or hyperglycemia-induced vascular injury is related to increasing NAMPT and NAD ${ }^{+}$followed by Sirt1mediated activation of AMPK/Sirt3 signals, thereby elevating cellular oxidation and decreasing apoptosis.

\section{Discussion}

In the present study, we demonstrated that acacetin antagonized hyperglycemic atherosclerosis with association of an improved intimal thickness increase of aortic arch and carotid artery distensibility and a reduced stenosis of carotid arteries, and an attenuated vascular lesion progression without decreasing blood glucose in STZ-diabetic $\mathrm{ApoE}^{-/-}$mice. The vascular protection of acacetin against high glucose insult is related to ameliorating mitochondrial oxidative stress and mitochondrial dysfunction. Sirt1mediated activation of AMPK/Sirt3 signals are proved to play a key role in mediating endothelial protective and anti-atherosclerotic action of acacetin in STZ-diabetic $\mathrm{ApoE}^{-/-}$mice. Notably, this study is the first to demonstrate the anti-atherosclerotic effect and potential mechanisms of acacetin against hyperglycemic injury, suggesting that acacetin may be a potential therapeutic drug candidate for reducing cardiovascular complications in diabetic patients.

Our previous studies have reported that acacetin is effective in treating atrial fibrillation by selectively blocking atrial potassium currents $\left(\mathrm{I}_{\mathrm{Kur}}, \mathrm{I}_{\mathrm{KACh}}\right.$, and $\left.\mathrm{sK}_{\mathrm{Ca}}\right)[23,24,38,39]$, and is cardioprotective against ischemia/reperfusion injury via increasing AMPK/Nrf2 and anti-oxidation and inhibiting apoptosis and inflammation [25,26]. Other groups demonstrated that acacetin has anticancer [40, 41], anti-peroxidation [42] and anti-neuronal inflammation $[43,44]$ effects. In addition, acacetin may reduce E-selectin expression in endothelial cells by regulating MAP kinase [45]. The present study provides the novel information that acacetin provides significant vascular protection against atherosclerosis in STZ-diabetic ApoE $^{-/-}$mice by Sirt1-mediated activation of AMPK, Sirt3 and PGC-1a signals. 
It is generally recognized that atherosclerosis is a multifactorial vascular progressive disorder involving alteration of several cellular and molecular events in diabetes $[12,46]$. These include the impairment of mitochondrial morphology and function associated with downregulation of SOD, pAMPK and PCG-1a, etc., leading to decrease of ATP product and increase of ROS production $[12,46]$. In the present study, diabetic neointima hyperplasia and atherosclerotic lesions were associated with downregulation of signaling molecules (i.e. SOD, Bcl-2, PGC-1a, pAMPK, Sirt3 and Sirt1) in artery tissues in STZ-diabetic $\mathrm{ApoE}^{-/-}$mice and also in HUVECs cultured with high glucose medium.

There are seven human sirtuin isoforms (Sirt1-Sirt7) located in nucleus (Sirt1, Sirt6, and Sirt7), mitochondria (Sirt3, Sirt4, and Sirt5), or cytoplasm (Sirt2) that are activated by NAD ${ }^{+}$and NAMPT $[35,47]$. Sirt 1 is widely studied from crossroads of nutrient (energy) sensing to various adaptive pathways. Sirt3 located mainly in mitochondria regulates metabolism and oxidative stress $[48,49]$. The present study demonstrated that mitochondrial Sirt3 was reduced with association of decrease of Sirt1, pAMPK and PGC-1a in artery tissues in STZ-diabetic $\mathrm{ApoE}^{-/-}$mice and also in high glucose-induced injury in cultured HUVECs. It appears that Sirt3 plays a crucial role in acacetin-mediated regulation of mitochondrial function including oxidation balance and ATP generation. Silencing Sirt3 abolishes the protective effect of acacetin against high glucose-induced ROS production and apoptosis, and ATP reduction in HUVECs, but does not affect acacetin-induced upregulation of Sirt1, PAMPK, and PGC-1a. This suggests that Sirt3 is not involved in regulation of Sirt1, pAMPK and PGC-1a.

However, silencing Sirt1 significantly decreased expression of Sirt3, pAMPK and PGC-1a and completely abolishes acacetin-induced upregulation of these molecules in HUVECs cultured with high glucose medium, whereas the AMPK inhibitor Compound $\mathrm{C}$ reduced expression of Sirt3, pAMPK and PGC-1 $\mathrm{a}$ and fully inhibited acacetin-induced upregulation of pAMPK and PGC-1a (partially inhibited Sirt3 upregulation by acacetin). AMPK inhibition does not affect Sirt1 expression or acacetin-induced upregulation. These results indicate that protection of acacetin against vascular hyperglycemic injury is related to Sirt1mediated activation of Sirt3 and AMPK,followed by AMPK-dependent PGC-1 a activation for regulating biosynthesis $[50,51]$, thereby slowing down atherosclerotic progression via inhibiting oxidation and apoptosis in STZ-diabetic $\mathrm{ApoE}^{-/-}$mice.

The clinical drug metformin [22] and a number of natural bioactive compounds [52-54] have been reported to have anti-atherosclerotic effect, including curcumin, quercetin, puerarin, resveratrol, etc. by activating Sirt1 and/or AMPK. However, most of these promising natural compounds face a problem of druggability due to the poor solubility or bioavailability. The present study showed that acacetin, in addition to activating PAMPK as previously reported [26], stimulates Sirt1, Sirt3, and PGC-1a and therefore is a novel activator of Sirt 1 with lower concentration range $(0.3-3 \mu \mathrm{M})$ than other previously reported activators with similar activation mechanism, i.e. increasing $\mathrm{NAD}^{+} / \mathrm{NADH}$ ratio. Importantly, the solubility and bioavailability issues of acacetin have been solved by synthesis of the water-soluble prodrug, which can be used clinically in the future to not only treat atrial fibrillation, myocardial ischemia/reperfusion injury, but also diabetic atherosclerosis. 
We observed that acacetin reversed the alterations of lipid profiles in STZ-diabetic ApoE ${ }^{-/-}$mice, including the decrease of the elevated triglyceride, total cholesterol, low-density lipoprotein, lipoprotein A, and lipoprotein $B$ and the increase of reduced high-density lipoprotein; the related mechanisms of these phenomena were not explored in the present study, and is a study limitation which remains clarified in the future. However, this limitation will not affect the conclusion of the present study.

\section{Conclusions}

This study demonstrates that novel information that acacetin treatment effectively lessens atherosclerosis, enhances the viability of endothelial cells and preserves mitochondrial function in STZdiabetic mice by Sirt1-mediated activation of AMPK/Sirt3 signaling molecules. These results indicate that acacetin treatment is likely a promising therapeutic drug candidate for slowing down the development of atherosclerosis in patients with diabetes.

\section{Abbreviations}

AMPK: Adenosine monophosphate-activated protein kinase; ApoE: Apolipoprotein E; Aden-osine triphosphate; Bax: Bcl2 associated X protein; Bcl2: B-cell lymphoma-2; $\beta \mathrm{OHB}$ : $\beta$-hydroxybutyrate; FBG Fasting blood glucose; HDL: High-density lipoprotein; HUVECs: Human umbilical vein endothelial cells; LCCA EDV: left common carotid artery end-diastolic velocity; LCCA PSV: left common carotid artery peak systolic velocity; LDL: Low-density lipoprotein; LP (A): Lipoprotein A; LP (B): Lipoprotein B; NAD+: Nicotinamide adenine dinucleotide, oxidized form; NADH: Nicotinamide adenine dinucleotide, reduced form; PGC-1a: Peroxisome proliferator-activated receptor-y coactivator-1a; RBG: Random blood glucose; RCCA EDV: right common carotid artery end-diastolic velocity; RCCA PSV: right common carotid artery peak systolic velocity; Sirt1: Sirtuin-1; Sirt3: Sirtuin-3; SOD1: Superoxide dismutase-1; SOD2: Superoxide dismutase-2; STZ: Streptozotocin; TC: Total cholesterol; TG: Triglyceride.

\section{Declarations}

\section{Acknowledgments}

This study was supported in part by a Science and Technology Cooperation Fund (U1605226) across the Taiwan Straits of the National Natural Science Foundation of China.

\section{Consent for publication}

All authors gave their consent for publication. 
The authors declare no conflict of interest.

\section{Authors Contributions}

WMH, GRL and YW designed the study. WMH and XCC acquired the experimental data. YW provided the financial support. WMH and GRL analyzed the data. WMH draft the manuscript, GRL revised the article critically for important intellectual content. All authors read and approved the final manuscript.

\section{Funding}

Science and Technology Cooperation Fund across Taiwan Straits of the National Natural Science Fundation of China (CN)(U1605226).

\section{Availability of data and materials}

Not applicable.

\section{Ethics approval and consent to participate}

The experimental protocol was reviewed and approved by Xiamen University Medical College(XMULAC20190120).

\section{Author details}

${ }^{1}$ Institute of Cardiovascular Disease, Xiamen University Medical College, Fujian 361000, China. ${ }^{2}$ Institute of Cardiovascular Disease, Xiamen University Medical College, Fujian 361000, China. ${ }^{3}$ Xiamen Cardiovascular Hospital, Xiamen University, Fujian 361000, China. ${ }^{4}$ Institute of Cardiovascular Disease, Xiamen University Medical College, Fujian 361000, China.

\section{References}

1. Beckman JA, Creager MA, Libby P: Diabetes and atherosclerosis: epidemiology, pathophysiology, and management. JAMA 2002, 287(19):2570-2581.

2. Nathan DMea: Intensive Diabetes Treatment and Cardiovascular Disease in Patients with Type 1 Diabetes\r10.1056/NEJMoa052187. New England Journal of Medicine 2005, 353(25):2643-2653. 
3. Orchard TJ, Costacou T, Kretowski A, Nesto RW: Type 1 diabetes and coronary artery disease. Diabetes Care 2006, 29(11):2528-2538.

4. Bianchi C, Miccoli R, Daniele G, Penno G, Del Prato S: Is there evidence that oral hypoglycemic agents reduce cardiovascular morbidity/mortality? Yes. Diabetes Care 2009, 32 Suppl 2:S342-348.

5. Poon AK, Meyer ML, Tanaka H, Selvin E, Pankow J, Zeng D, Loehr L, Knowles JW, Rosamond W, Heiss G: Association of insulin resistance, from mid-life to late-life, with aortic stiffness in late-life: the Atherosclerosis Risk in Communities Study. Cardiovascular diabetology 2020, 19(1):11.

6. Takahara M, lida O, Kohsaka S, Soga Y, Fujihara M, Shinke T, Amano T, Ikari Y, J EVT, investigators JP: Diabetes mellitus and other cardiovascular risk factors in lower-extremity peripheral artery disease versus coronary artery disease: an analysis of 1,121,359 cases from the nationwide databases. Cardiovascular diabetology 2019, 18(1):155.

7. Paneni F, Beckman JA, Creager MA, Cosentino F: Diabetes and vascular disease: pathophysiology, clinical consequences, and medical therapy: part I. Eur Heart J 2013, 34(31):2436-2443.

8. Beckman JA, Paneni F, Cosentino F, Creager MA: Diabetes and vascular disease: pathophysiology, clinical consequences, and medical therapy: part II. Eur Heart J 2013, 34(31):2444-2452.

9. Juutilainen A, Lehto S, Ronnemaa T, Pyorala K, Laakso M: Similarity of the impact of type 1 and type 2 diabetes on cardiovascular mortality in middle-aged subjects. Diabetes Care 2008, 31(4):714-719.

10. Kanter JE, Johansson F, LeBoeuf RC, Bornfeldt KE: Do glucose and lipids exert independent effects on atherosclerotic lesion initiation or progression to advanced plaques? Circ Res 2007, 100(6):769781.

11. Nagareddy PR, Murphy AJ, Stirzaker RA, Hu Y, Yu S, Miller RG, Ramkhelawon B, Distel E, Westerterp M, Huang LS et al: Hyperglycemia promotes myelopoiesis and impairs the resolution of atherosclerosis. Cell Metab 2013, 17(5):695-708.

12. Madonna R, Pieragostino D, Balistreri CR, Rossi C, Geng YJ, Del Boccio P, De Caterina R: Diabetic macroangiopathy: Pathogenetic insights and novel therapeutic approaches with focus on high glucose-mediated vascular damage. Vascular pharmacology 2018.

13. Bornfeldt KE: 2013 Russell Ross memorial lecture in vascular biology: cellular and molecular mechanisms of diabetes mellitus-accelerated atherosclerosis. Arterioscler Thromb Vasc Biol 2014, 34(4):705-714.

14. Calkin AC, Allen TJ: Diabetes mellitus-associated atherosclerosis: mechanisms involved and potential for pharmacological invention. Am J Cardiovasc Drugs 2006, 6(1):15-40.

15. Gray SP, Di Marco E, Okabe J, Szyndralewiez C, Heitz F, Montezano AC, de Haan JB, Koulis C, El-Osta $\mathrm{A}$, Andrews $\mathrm{KL}$ et al: NADPH oxidase 1 plays a key role in diabetes mellitus-accelerated atherosclerosis. Circulation 2013, 127(18):1888-1902.

16. Giacco F, Brownlee M: Oxidative stress and diabetic complications. Circ Res 2010, 107(9):1058-1070.

17. Rao G, Ding HG, Huang W, Le D, Maxhimer JB, Oosterhof A, van Kuppevelt T, Lum H, Lewis EJ, Reddy $\checkmark$ et al: Reactive oxygen species mediate high glucose-induced heparanase-1 production and 
heparan sulphate proteoglycan degradation in human and rat endothelial cells: a potential role in the pathogenesis of atherosclerosis. Diabetologia 2011, 54(6):1527-1538.

18. Yuan T, Yang T, Chen H, Fu D, Hu Y, Wang J, Yuan Q, Yu H, Xu W, Xie X: New insights into oxidative stress and inflammation during diabetes mellitus-accelerated atherosclerosis. Redox Biol 2019, 20:247-260.

19. Sharma A, Sellers S, Stefanovic N, Leung C, Tan SM, Huet O, Granville DJ, Cooper ME, de Haan JB, Bernatchez P: Direct Endothelial Nitric Oxide Synthase Activation Provides Atheroprotection in Diabetes-Accelerated Atherosclerosis. Diabetes 2015, 64(11):3937-3950.

20. Pratico D: Antioxidants and endothelium protection. Atherosclerosis 2005, 181(2):215-224.

21. Ding H, Hashem M, Wiehler WB, Lau W, Martin J, Reid J, Triggle C: Endothelial dysfunction in the streptozotocin-induced diabetic apoE-deficient mouse. Br J Pharmacol 2005, 146(8):1110-1118.

22. Wang Q, Zhang M, Torres G, Wu S, Ouyang C, Xie Z, Zou MH: Metformin Suppresses DiabetesAccelerated Atherosclerosis via the Inhibition of Drp1-Mediated Mitochondrial Fission. Diabetes 2017, 66(1):193-205.

23. Li GR, Wang HB, Qin GW, Jin MW, Tang Q, Sun HY, Du XL, Deng XL, Zhang XH, Chen JB et al: Acacetin, a natural flavone, selectively inhibits human atrial repolarization potassium currents and prevents atrial fibrillation in dogs. Circulation 2008, 117(19):2449-2457.

24. Liu H, Wang YJ, Yang L, Zhou M, Jin MW, Xiao GS, Wang Y, Sun HY, Li GR: Synthesis of a highly water-soluble acacetin prodrug for treating experimental atrial fibrillation in beagle dogs. Scientific reports 2016, 6:25743.

25. Liu H, Yang L, Wu HJ, Chen KH, Lin F, Li G, Sun HY, Xiao GS, Wang Y, Li GR: Water-soluble acacetin prodrug confers significant cardioprotection against ischemia/reperfusion injury. Scientific reports $2016,6: 36435$.

26. Wu WY, Li YD, Cui YK, Wu C, Hong YX, Li G, Wu Y, Jie LJ, Wang Y, Li GR: The Natural Flavone Acacetin Confers Cardiomyocyte Protection Against Hypoxia/Reoxygenation Injury via AMPK-Mediated Activation of Nrf2 Signaling Pathway. Frontiers in pharmacology 2018, 9:497.

27. Guo Y, Liu XC, Wang YJ, Li Q, Yang Q, Weng XG, Chen Y, Cai WY, Kan XX, Chen X et al: Effects of Shenlian extract on experimental atherosclerosis in ApoE-deficient mice based on ultrasound biomicroscopy. BMC Complement Altern Med 2016, 16(1):469.

28. Dong Y, Zhang M, Liang B, Xie Z, Zhao Z, Asfa S, Choi HC, Zou MH: Reduction of AMP-activated protein kinase alpha2 increases endoplasmic reticulum stress and atherosclerosis in vivo. Circulation 2010, 121(6):792-803.

29. Kennedy DJ, Kuchibhotla SD, Guy E, Park YM, Nimako G, Vanegas D, Morton RE, Febbraio M: Dietary cholesterol plays a role in CD36-mediated atherogenesis in LDLR-knockout mice. Arterioscler Thromb Vasc Biol 2009, 29(10):1481-1487.

30. Ganguly R, Sahu S, Ohanyan V, Haney R, Chavez RJ, Shah S, Yalamanchili S, Raman P: Oral chromium picolinate impedes hyperglycemia-induced atherosclerosis and inhibits proatherogenic 
protein TSP-1 expression in STZ-induced type 1 diabetic ApoE(-/-) mice. Scientific reports 2017, 7:45279.

31. Yang Y, Duan W, Jin Z, Yi W, Yan J, Zhang S, Wang N, Liang Z, Li Y, Chen W et al: JAK2/STAT3 activation by melatonin attenuates the mitochondrial oxidative damage induced by myocardial ischemia/reperfusion injury. Journal of Pineal Research 2013, 55(3):275-286.

32. Blackshear WM, Phillips DJ, Chikos PM, Harley JD, Thiele BL, Strandness DE, Jr.: Carotid artery velocity patterns in normal and stenotic vessels. Stroke 1980, 11(1):67-71.

33. Low Wang CC, Hess CN, Hiatt WR, Goldfine AB: Clinical Update: Cardiovascular Disease in Diabetes Mellitus: Atherosclerotic Cardiovascular Disease and Heart Failure in Type 2 Diabetes Mellitus Mechanisms, Management, and Clinical Considerations. Circulation 2016, 133(24):2459-2502.

34. Shah MS, Brownlee M: Molecular and Cellular Mechanisms of Cardiovascular Disorders in Diabetes. Circulation research 2016, 118(11):1808-1829.

35. Imai SI, Guarente L: It takes two to tango: $\mathrm{NAD}(+)$ and sirtuins in aging/longevity control. NPJ aging and mechanisms of disease 2016, 2:16017.

36. Anderson KA, Madsen AS, Olsen CA, Hirschey MD: Metabolic control by sirtuins and other enzymes that sense $\mathrm{NAD}(+), \mathrm{NADH}$, or their ratio. Biochimica et biophysica acta Bioenergetics 2017, 1858(12):991-998.

37. Cerna D, Li H, Flaherty S, Takebe N, Coleman CN, Yoo SS: Inhibition of nicotinamide phosphoribosyltransferase (NAMPT) activity by small molecule GMX1778 regulates reactive oxygen species (ROS)-mediated cytotoxicity in a p53- and nicotinic acid phosphoribosyltransferase 1 (NAPRT1)-dependent manner. The Journal of biological chemistry 2012, 287(26):22408-22417.

38. Wu HJ, Wu W, Sun HY, Qin GW, Wang HB, Wang P, Yalamanchili HK, Wang J, Tse HF, Lau CP et al: Acacetin causes a frequency- and use-dependent blockade of hKv1.5 channels by binding to the $S 6$ domain. Journal of molecular and cellular cardiology 2011, 51(6):966-973.

39. Chen KH, Liu H, Sun HY, Jin MW, Xiao GS, Wang Y, Li GR: The Natural Flavone Acacetin Blocks Small Conductance $\mathrm{Ca}(2+)$-Activated K(+) Channels Stably Expressed in HEK 293 Cells. Frontiers in pharmacology 2017, 8:716.

40. Fong Y, Shen KH, Chiang TA, Shih YW: Acacetin inhibits TPA-induced MMP-2 and u-PA expressions of human lung cancer cells through inactivating JNK signaling pathway and reducing binding activities of NF-kappaB and AP-1. J Food Sci 2010, 75(1):H30-38.

41. Kim HR, Park CG, Jung JY: Acacetin (5,7-dihydroxy-4'-methoxyflavone) exhibits in vitro and in vivo anticancer activity through the suppression of NF-kappaB/Akt signaling in prostate cancer cells. Int $J$ Mol Med 2014, 33(2):317-324.

42. M.R.Cholbi MP, M.J.Alcaraz: Inhibitory effects of phenolic compounds on CCl4-induced microsomal lipid peroxidation. Experientia 1991, 47 195-199.

43. Yin $Y$, Gong $F Y$, Wu XX, Sun Y, Li YH, Chen T, Xu Q: Anti-inflammatory and immunosuppressive effect of flavones isolated from Artemisia vestita. J Ethnopharmacol 2008, 120(1):1-6. 
44. Bu J, Shi S, Wang HQ, Niu XS, Zhao ZF, Wu WD, Zhang XL, Ma Z, Zhang YJ, Zhang H et al: Acacetin protects against cerebral ischemia-reperfusion injury the NLRP3 signaling pathway. Neural Regen Res 2019, 14(4):605-612.

45. Tanigawa N, Hagiwara M, Tada H, Komatsu T, Sugiura S, Kobayashi K, Kato Y, Ishida N, Nishida K, Ninomiya $\mathrm{M}$ et al: Acacetin inhibits expression of E-selectin on endothelial cells through regulation of the MAP kinase signaling pathway and activation of NF-KB. Immunopharmacol Immunotoxicol 2013, 35(4):471-477.

46. Gregg EW, Sattar N, Ali MK: The changing face of diabetes complications. Lancet Diabetes Endocrinol 2016, 4(6):537-547.

47. Wang Y, He J, Liao M, Hu M, Li W, Ouyang H, Wang X, Ye T, Zhang Y, Ouyang L: An overview of Sirtuins as potential therapeutic target: Structure, function and modulators. European journal of medicinal chemistry 2019, 161:48-77.

48. Yu L, Gong B, Duan W, Fan C, Zhang J, Li Z, Xue X, Xu Y, Meng D, Li B et al: Melatonin ameliorates myocardial ischemia/reperfusion injury in type 1 diabetic rats by preserving mitochondrial function: role of AMPK-PGC-1alpha-SIRT3 signaling. Scientific reports 2017, 7:41337.

49. Gallardo-Montejano VI, Saxena G, Kusminski CM, Yang C, McAfee JL, Hahner L, Hoch K, Dubinsky W, Narkar VA, Bickel PE: Nuclear Perilipin 5 integrates lipid droplet lipolysis with PGC-1alpha/SIRT1dependent transcriptional regulation of mitochondrial function. Nat Commun 2016, 7:12723.

50. Cantó C, Gerhart-Hines Z, Feige JN, Lagouge M, Noriega L, Milne JC, Elliott PJ, Puigserver P, Auwerx J: AMPK regulates energy expenditure by modulating NAD+ metabolism and SIRT1 activity. Nature 2009, 458(7241):1056-1060.

51. Joseph AM, Joanisse DR, Baillot RG, Hood DA: Mitochondrial dysregulation in the pathogenesis of diabetes: potential for mitochondrial biogenesis-mediated interventions. Exp Diabetes Res 2012, 2012:642038.

52. Moss JW, Ramji DP: Nutraceutical therapies for atherosclerosis. Nature reviews Cardiology 2016, 13(9):513-532.

53. Lin K, Chen H, Chen X, Qian J, Huang S, Huang W: Efficacy of Curcumin on Aortic Atherosclerosis: A Systematic Review and Meta-Analysis in Mouse Studies and Insights into Possible Mechanisms. Oxidative medicine and cellular longevity 2020, 2020:1520747.

54. Huminiecki L, Atanasov AG, Horbanczuk J: Etiology of atherosclerosis informs choice of animal models and tissues for initial functional genomic studies of resveratrol. Pharmacological research 2020:104598.

\section{Figures}


A

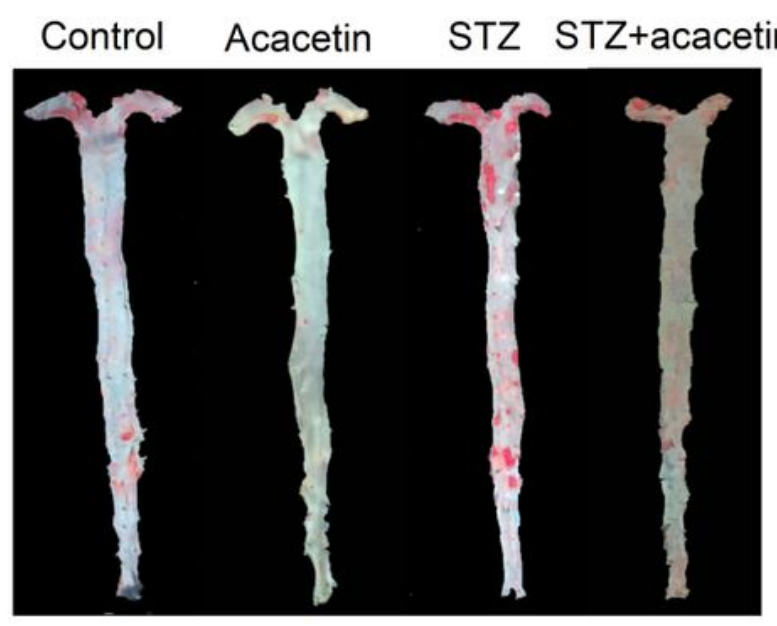

C

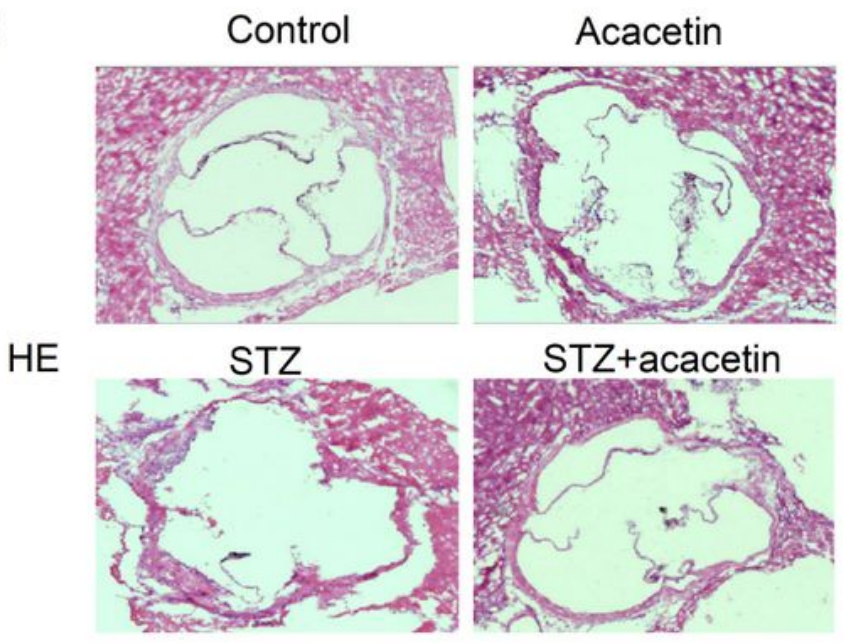

E

Oil

Red O

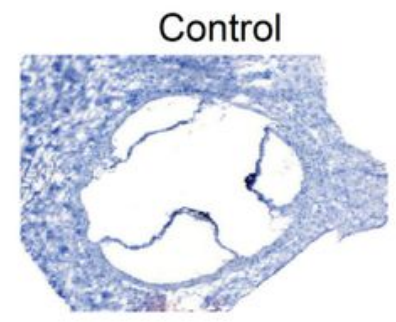

STZ

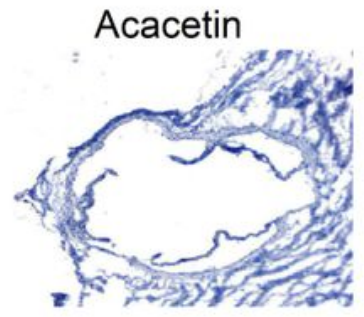

STZ+acacetin

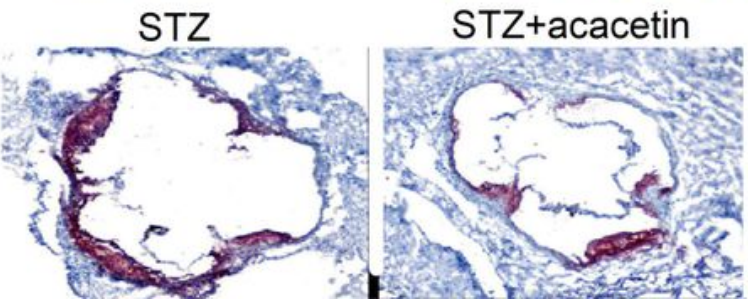

D

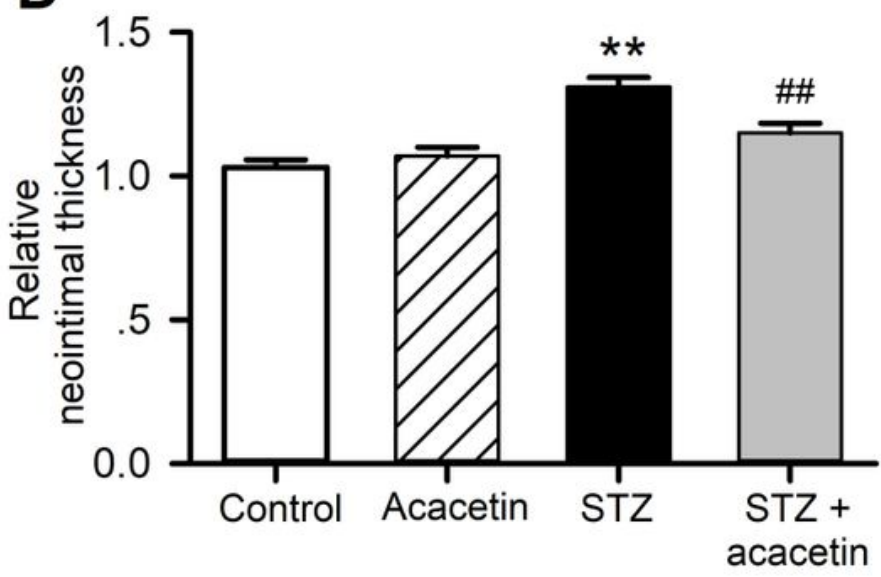

F

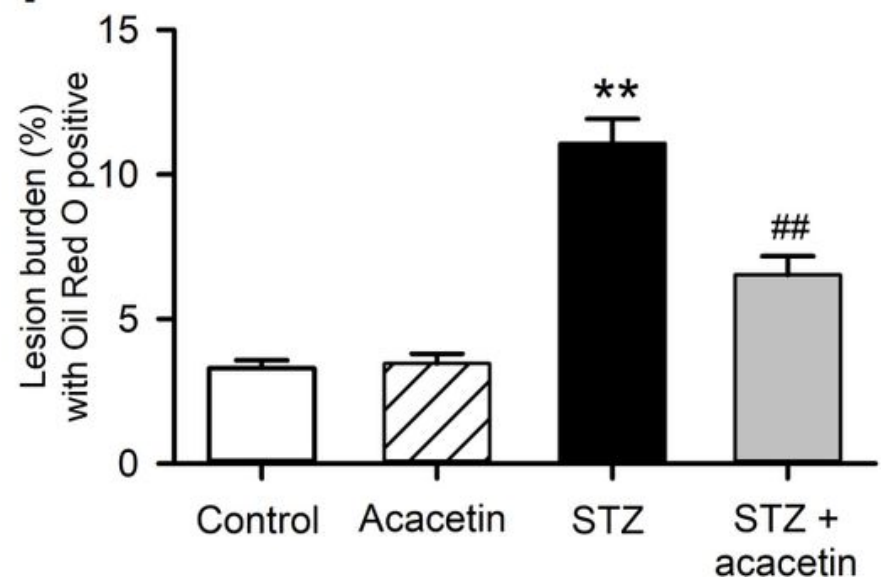

Figure 1

Acacetin attenuates STZ-induced atherosclerosis in STZ-diabetic ApoE-/- mice. (A) Oil Red O staining of mouse aortas in control, with acacetin treatment, STZ, and STZ with aca-cetin treatment. (B) Analysis of aorta lesion areas stained with Oil Red $\mathrm{O}$ in A. (C) HE stain-ing of aortic root section from mice in control, with acacetin, STZ, and STZ with acacetin treatment. (D) Analysis of neointimal thickness in C. (E) Oil Red $\mathrm{O}$ staining of aortic root sections from mice as in C. (F) Lesion burden of aortic root section with Oil 
Red 0 positive in $E$ (4X magnification). $N=11 * P<0.05, * * P<0.01$ vs. control or acacetin; \#P<0.05, \#\#P<0.01 vs. STZ.

A
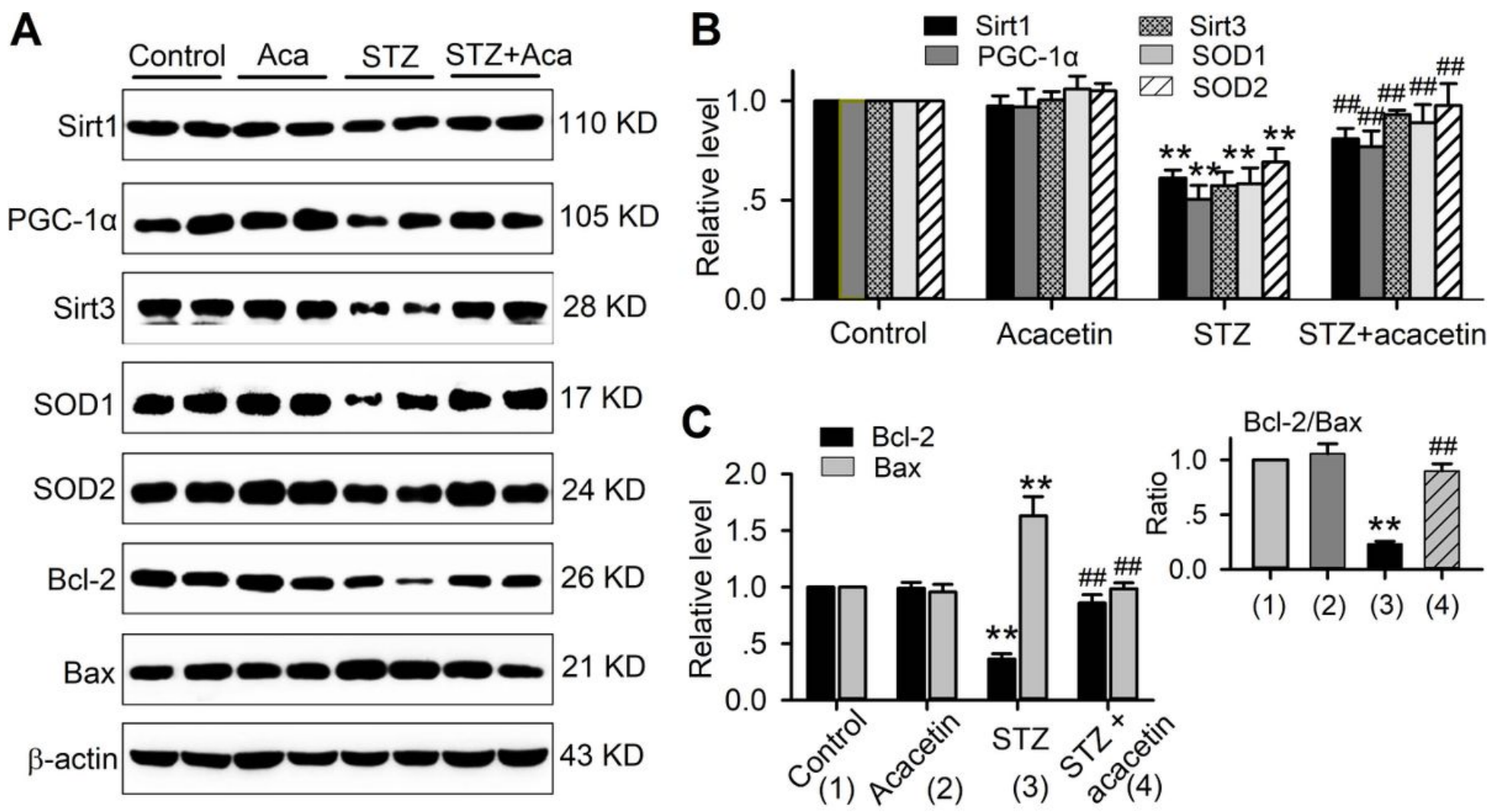

D
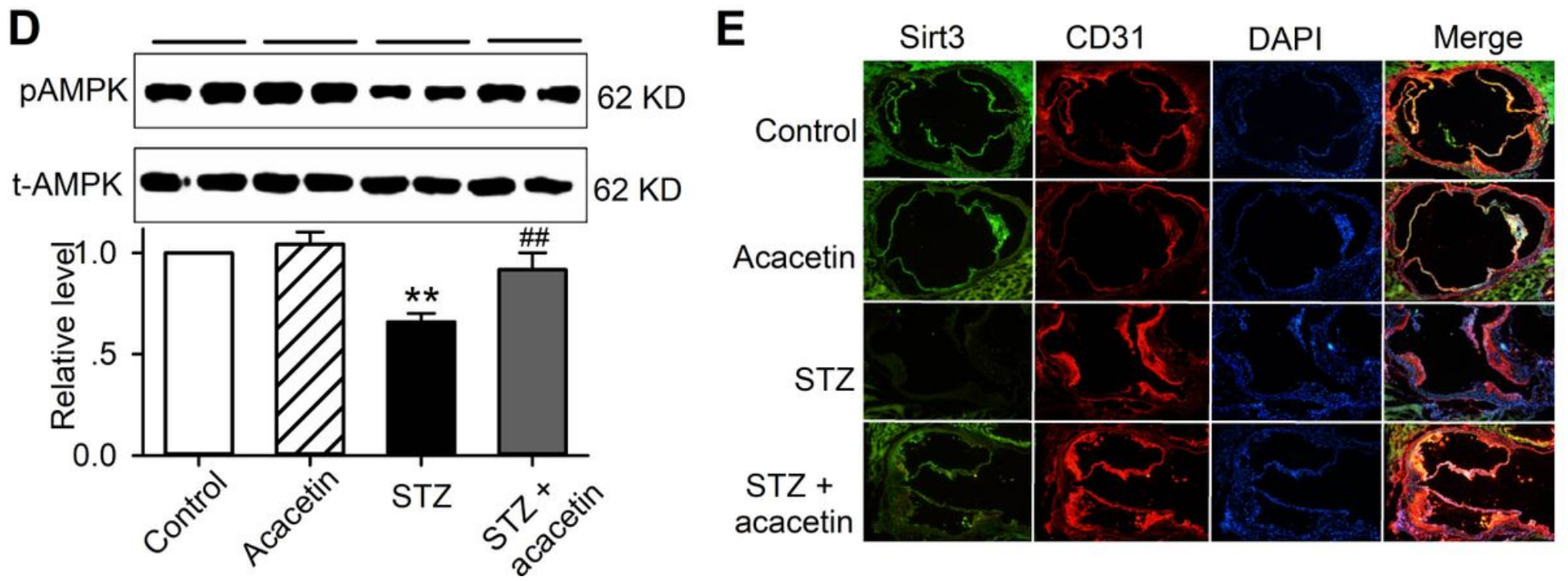

Figure 2

Alterations of related proteins in aortic tissues of STZ-diabetic ApoE-/- mice. (A) Western blots of Sirt1, PGC-1a, Sirt3, SOD1, SOD2, Bcl2, Bax, and $\beta$-actin. (B) Analysis of Sirt1, PGC-1a, Sirt3, SOD1, and SOD2 (relative to $\beta$-actin). (C) Analysis of $\mathrm{Bcl}-2$ and Bax proteins, relative to $\beta$-actin. Inset: ratio of $\mathrm{Bcl}-2 / \mathrm{Bax}$. (D) Western blots of pAMPK and t-AMPK (total AMPK) and relative level of pAMPK/t-AMPK. $N=6,{ }^{*}<<0.05$, ${ }^{\star} * P<0.01$ vs. control or aca-cetin; \#P<0.05, \#\#P<0.01 vs. STZ. (E) Sirt3 and CD31 immunohistochemical 
staining of aortic root section from mice of control, with acacetin, STZ, and STZ with acacetin treatment (10X magnification).
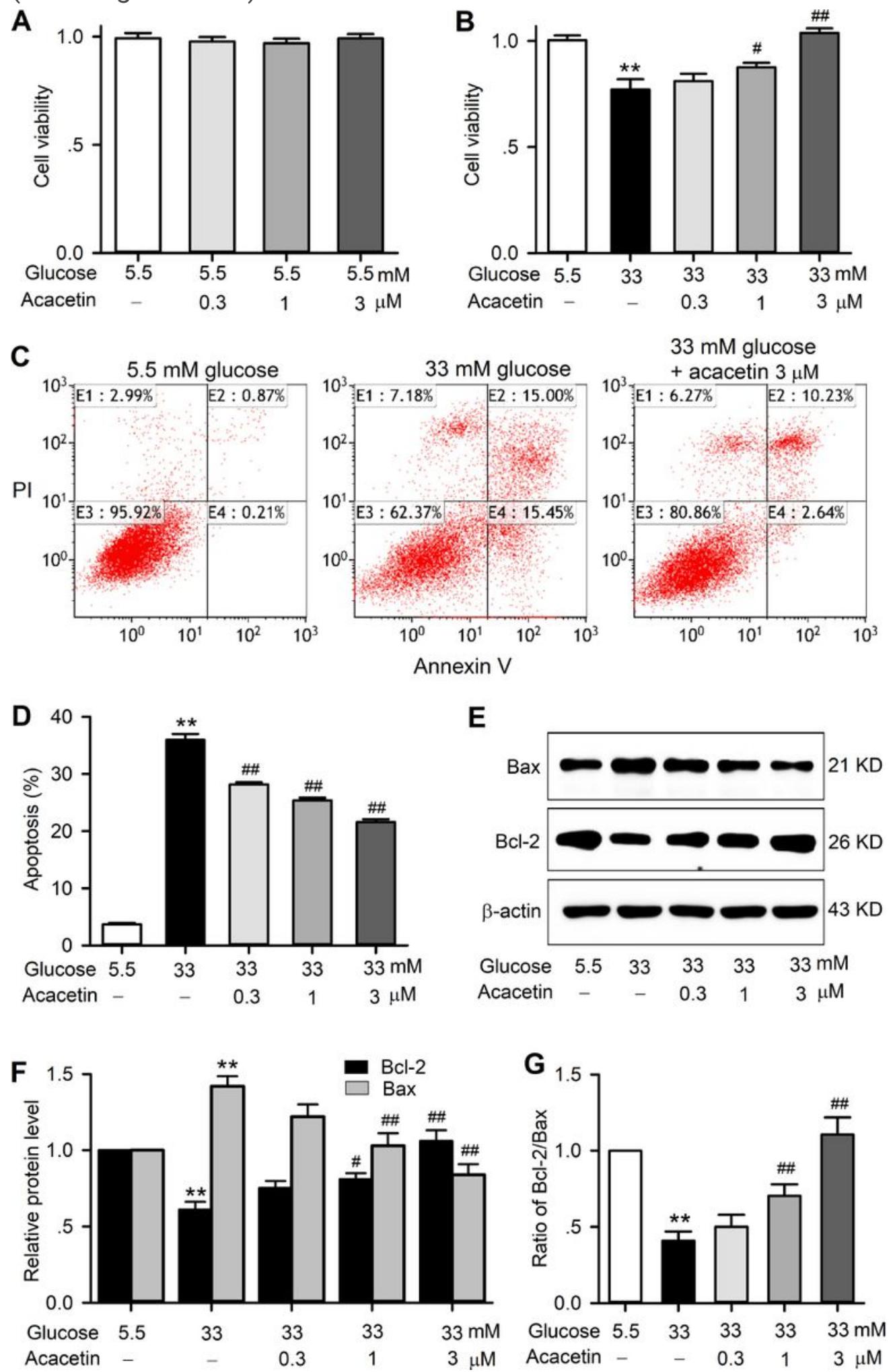

Figure 3

Effects of acacetin on cell activity and apoptosis induced by high glucose exposure in HUVECs. (A) Acacetin had no effect on cell viability in HUVECs cultured with $5.5 \mathrm{mM}$ glucose medium. (B) Acacetin countered viability reduction induced by high glucose medium culture in a concentration-dependent 
manner. (C) Flow cytometry graphs for cell apoptosis (E2+E4) in cells cultured with $5.5 \mathrm{mM}$ or $33 \mathrm{mM}$ glucose medium, or $33 \mathrm{mM}$ glucose plus $3 \mu \mathrm{M}$ acacetin. (D) Acacetin decreased apoptosis induced by high glucose culture in a concen-tration-dependent manner. (E) Western blots of apoptosis-related proteins Bax and Bcl-2 in HUVECs culture with $5.5 \mathrm{mM}$ glucose $(5.5 \mathrm{mM})$ or $33 \mathrm{mM}$ high glucose, or 33 $\mathrm{mM}$ glucose with $0.3,1$ and $3 \mu \mathrm{M}$ acacetin. $(\mathrm{F})$ Acacetin reversed the Bax increase and Bcl-2 decrease by high glucose culture in a concentration-dependent manner. (G) Acacetin countered the de-creased ratio of $\mathrm{Bcl}-2 / \mathrm{Bax}$ in a concentration-dependent manner. $\mathrm{N}=5,{ }^{*} \mathrm{P}<0.05,{ }^{*} \mathrm{P}<0.01 \mathrm{vs}$. $5.5 \mathrm{mM}$ glucose; $\# \mathrm{P}<0.05$, $\# \#$ P $<0.01$ vs. $33 \mathrm{mM}$ glucose). 
A

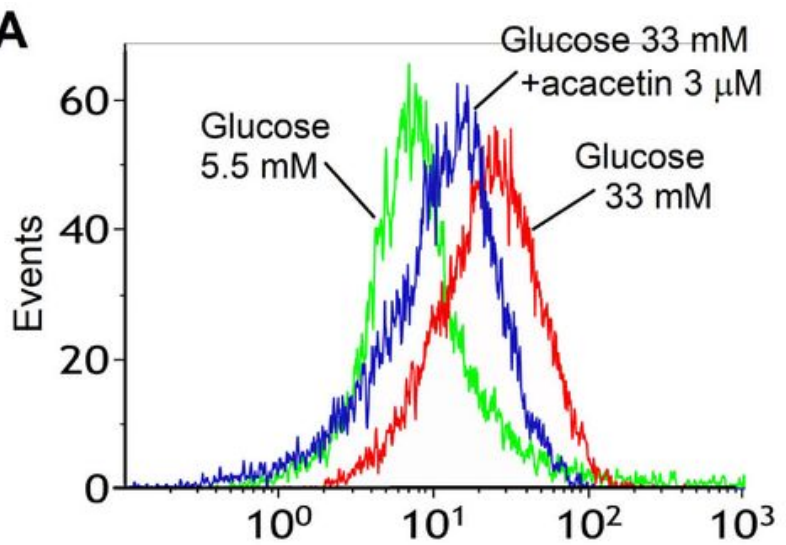

DCF-fluorescence

C

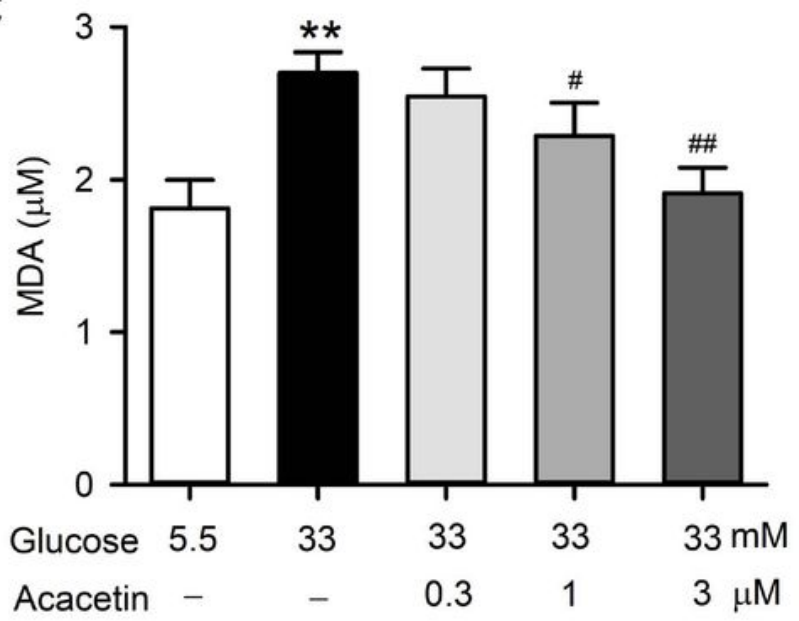

$\mathbf{E}$

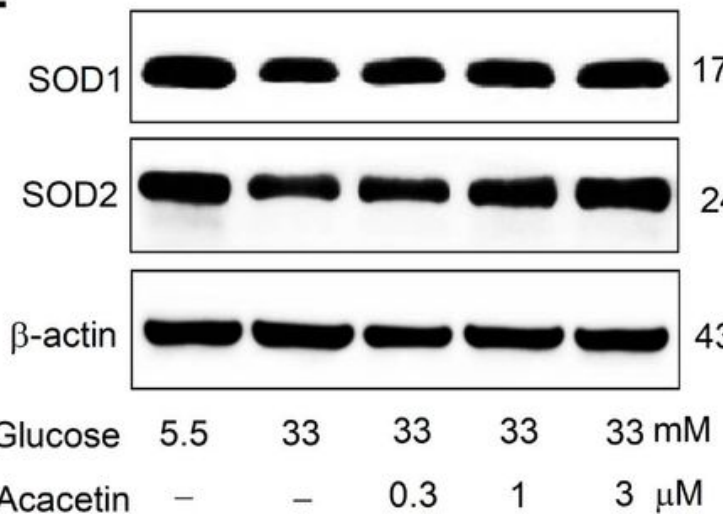

B

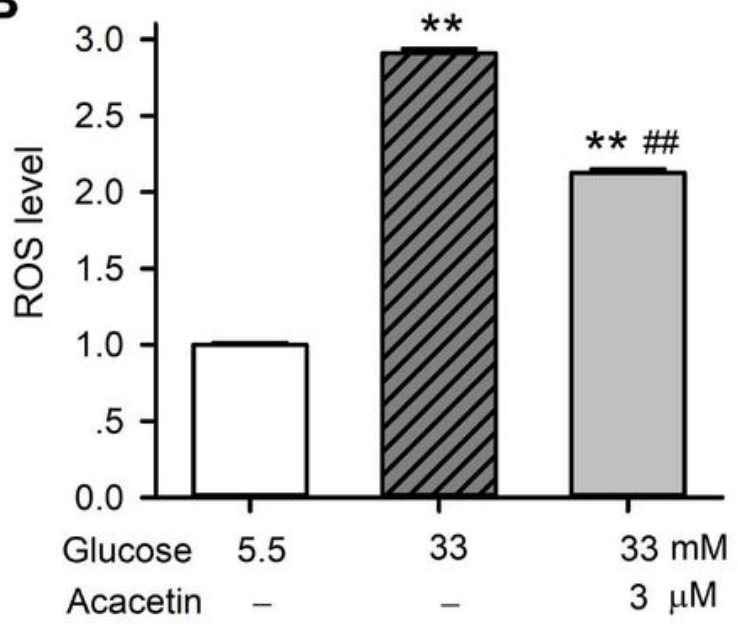

D

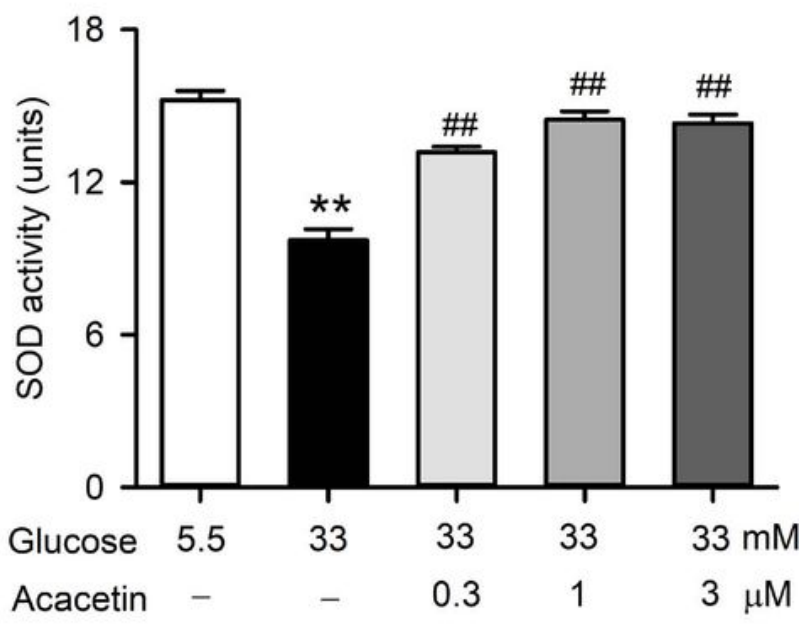

Figure 4

Effects of acacetin on ROS production and alteration of superoxide dismutase in HU-VECs induced by high glucose exposure. (A) Flow cytometry graphs of ROS production in HUVECs cultured with $5.5 \mathrm{mM}$ glucose medium, $33 \mathrm{mM}$ glucose medium or $33 \mathrm{mM}$ glucose medium plus $3 \mu \mathrm{M}$ acacetin. (B) Acacetin decreased high glucose-induced ROS increase. (C) Acacetin decreased high glucose-induced MDA increase in a concentration-dependent man-ner. (D) Acacetin reversed high glucose-induced decrease in 
SOD activity measured by the commercial kit in a concentration-dependent manner. (E) Western blots of SOD1 and SOD2 in HUVECs cultured with $5.5 \mathrm{mM}$ glucose medium, $33 \mathrm{mM}$ glucose medium or $33 \mathrm{mM}$ glu-cose medium plus acacetin. (F) Acacetin reversed high glucose-induced reduction of SOD1 and SOD2 proteins in a concentration-dependent manner. $N=5,{ }^{*} P<0.05,{ }^{\star \star} P<0.01$ vs. $5.5 \mathrm{mM}$ glucose; $\# P<0.05$, \#\#P<0.01 vs. 33 mM glucose).

A

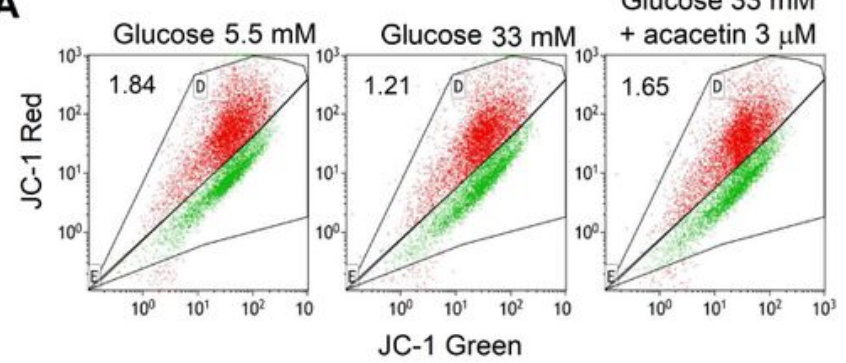

B

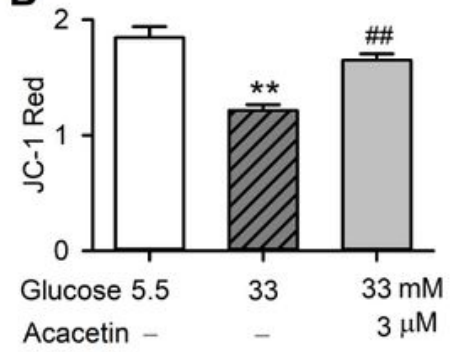

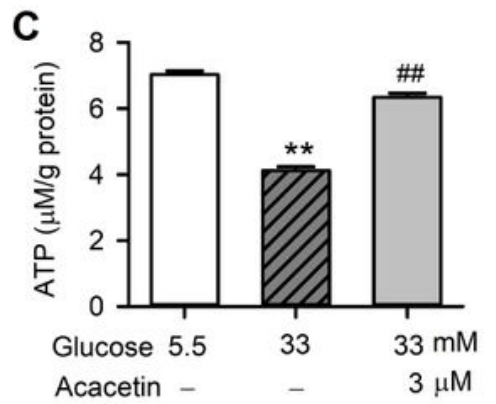
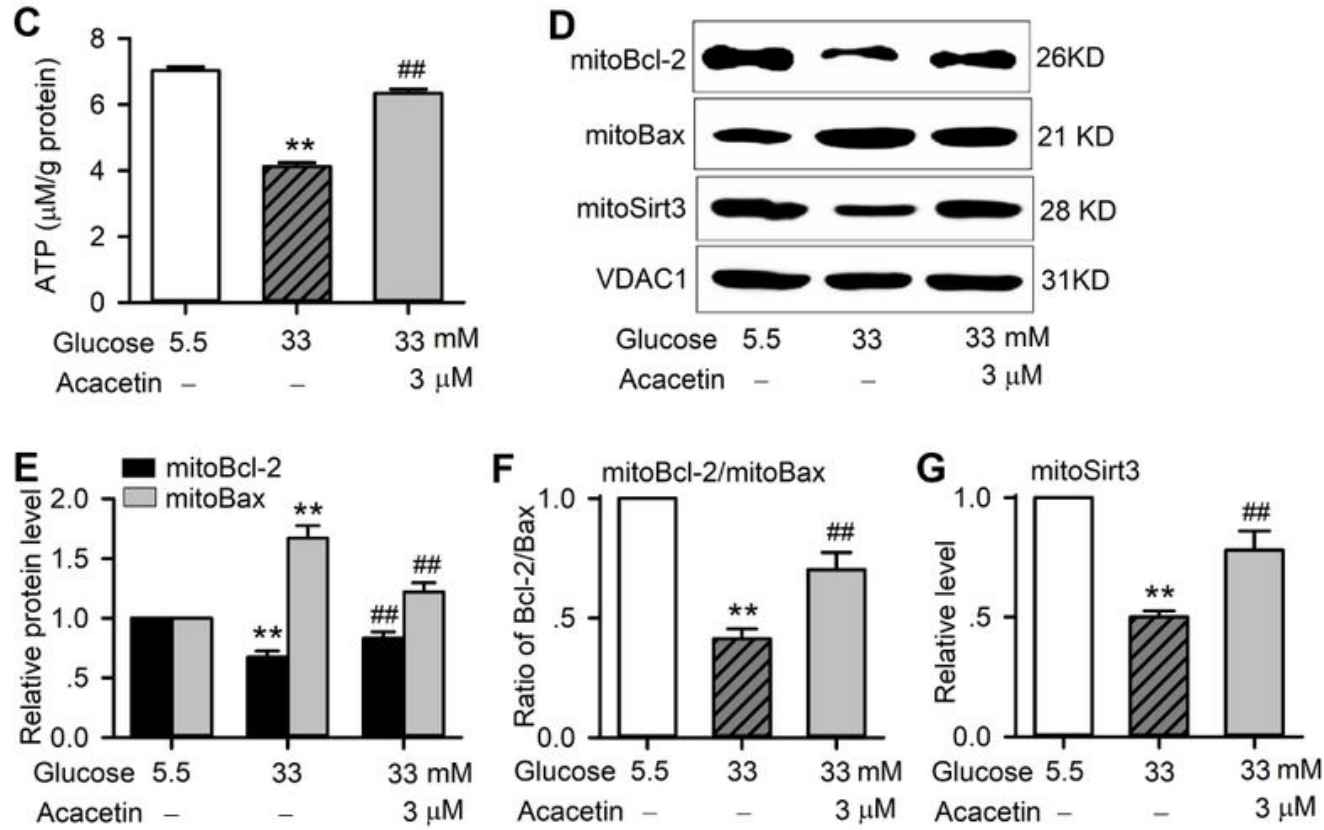

H

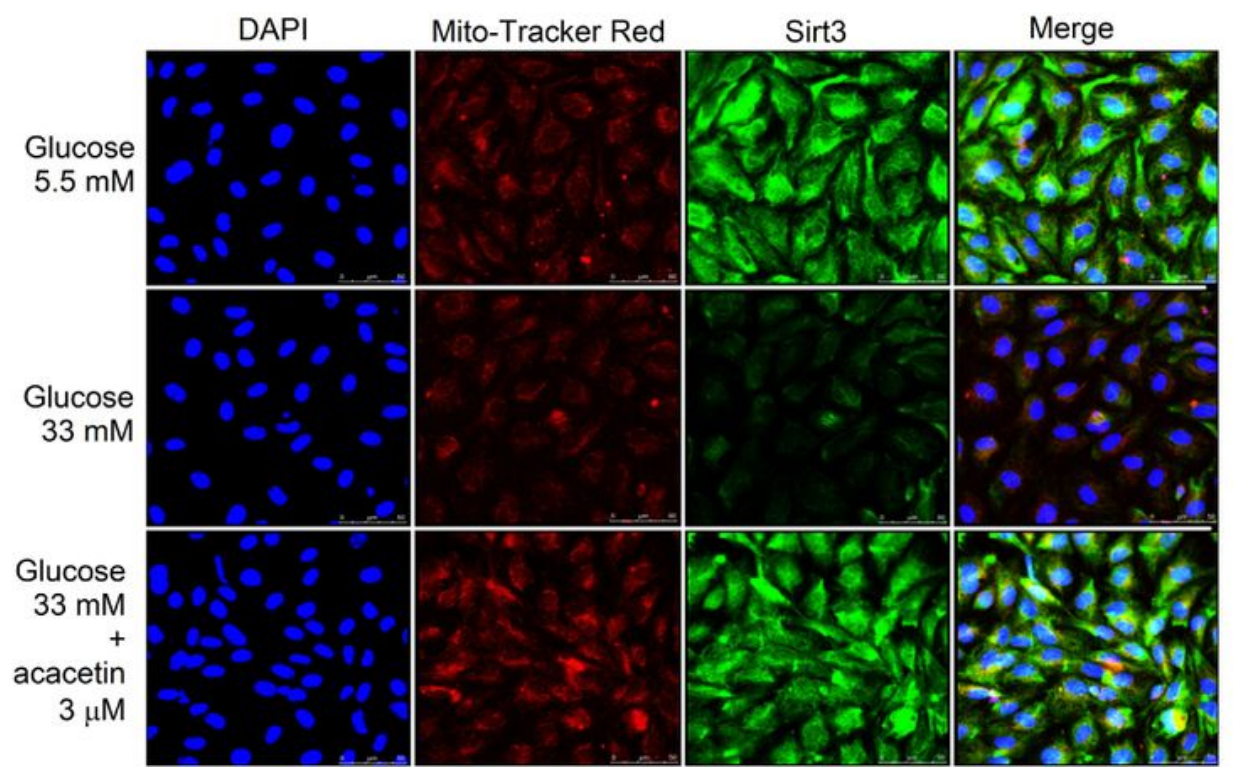

Figure 5 
Protective effects of acacetin on mitochondrial dysfunction and injury induced by high glucose exposure in HUVECs. (A) Flow cytometry graphs for determining mitochondrial transmembrane potential in HUVECs cultured with $5.5 \mathrm{mM}$ glucose medium, $33 \mathrm{mM}$ glucose medium or $33 \mathrm{mM}$ glucose medium plus $3 \mu \mathrm{M}$ acacetin. (B) Acacetin reversed the decrease of mitochondrial transmembrane potential in cells with high glucose exposure. (C) Acacetin countered high glucose-induced decrease of ATP production. (D) Western blots of mitochon-drial Bcl-2, Bax, and Sirt3 in cells cultured with $5.5 \mathrm{mM}$ glucose medium, 33 $\mathrm{mM}$ glucose medium or $33 \mathrm{mM}$ glucose medium plus acacetin. (E) Relative (to VDAC1, volt-agedependent anion channel 1) mitochondrial Bcl-2 and Bax protein levels. (F) Acacetin reversed the reduced ratio of mitochondrial $\mathrm{Bcl}-2 / \mathrm{Bax}$ proteins. $(\mathrm{G})$ Acacetin upregulated high glucose-induced reduction of Sirt3 protein. $(\mathrm{H})$ Sirt3 immunocytochemical staining and mitoTracker Red co-staining (80X magnification) in HUVECs cultured with $5.5 \mathrm{mM}$ glucose medium, $33 \mathrm{mM}$ glucose medium or $33 \mathrm{mM}$ glucose medium plus $3 \mu \mathrm{M}$ acacetin. $N=5,{ }^{*}<<0.05,{ }^{\star *} P<0.01$ vs. $5.5 \mathrm{mM}$ glucose; $\# P<0.05, \# \# P<0.01$ vs. $33 \mathrm{mM}$ glucose. 

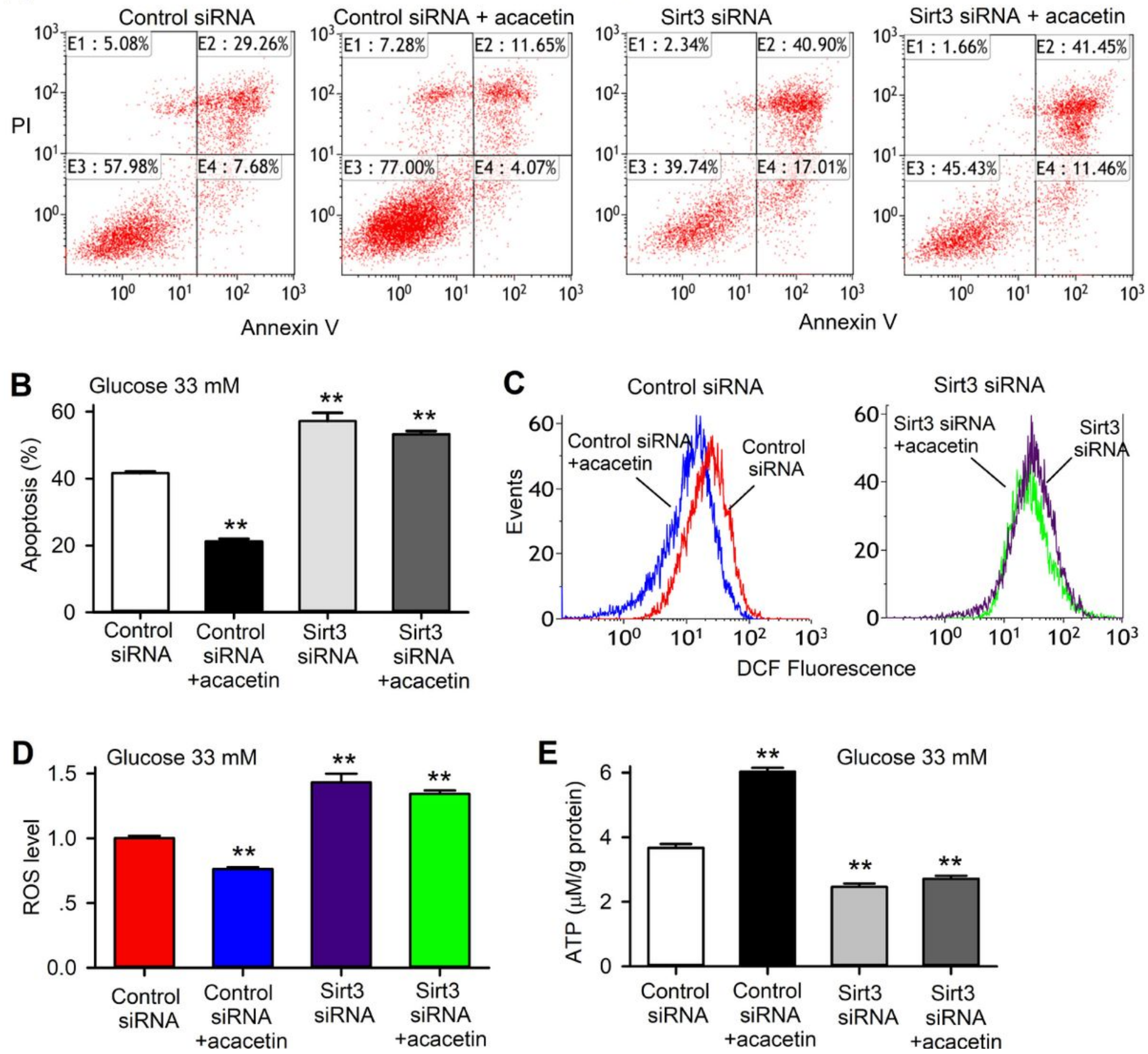

Figure 6

Sirt3 silencing and acacetin effects on apoptosis and ROS production induced by high glucose exposure in HUVECs. (A) Flow cytometry graphs for analyzing apoptosis (E2+E4) in cells transfected with control siRNA or Sirt3 siRNA and cultured with $33 \mathrm{mM}$ glucose me-dium in the absence or presence of $3 \mu \mathrm{M}$ acacetin. (B) Acacetin decreased apoptosis in cells transfected with control siRNA, but not in cells transfected with Sirt3 siRNA. (C) Flow cy-tometry graphs for determining ROS levels in cells transfected with control siRNA or Sirt3 siRNA and cultured with $33 \mathrm{mM}$ glucose medium in the absence or presence of $3 \mu \mathrm{M}$ aca-cetin. (D) Acacetin reduced ROS production in cells transfected with control siRNA, but not in 
cells transfected with Sirt3 siRNA. (E) Acacetin increased ATP in cells transfected with control siRNA, but not in cells transfected with Sirt3 siRNA. $N=5, * * P<0.01$ vs. control siRNA.
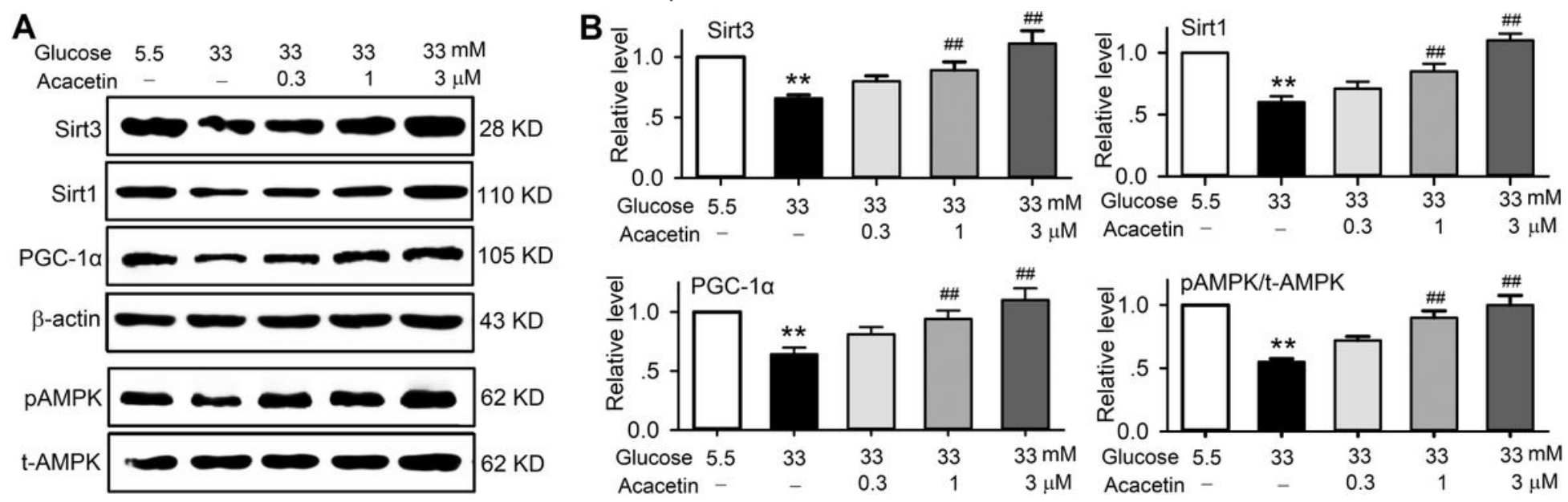

Figure 7

Acacetin rescued the impaired signal molecules induced by high glucose exposure in HUVECs. (A) Western blots of Sirt1, Sirt3, PGC-1a, pAMPK, t-AMPK in HUVECs cul-tured in 5.5 mM glucose or 33 mM glucose in the absence or presence of $0.3,1$, or $3 \mu \mathrm{M}$ aca-cetin. (B) Acacetin reversed the decrease of Sirt1, Sirt3, PGC-1 $a$ and pAMPK in a concentra-tion-dependent manner. $N=5$, ** $P<0.01$ vs. 5.5 mM glucose; \#\#P<0.01 vs. 33 mM glucose alone.

A Glucose $33 \mathrm{mM}$

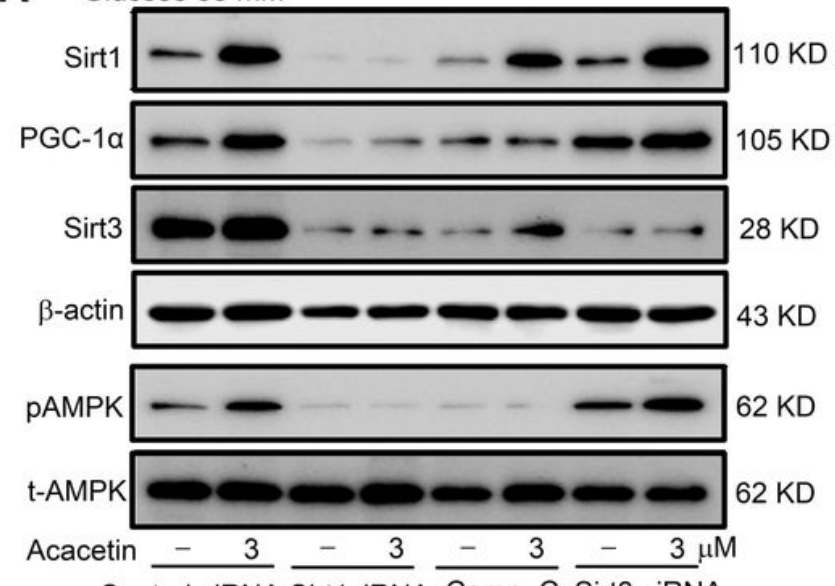

Control siRNA Sirt1siRNA Comp. C Sirt3 siRNA
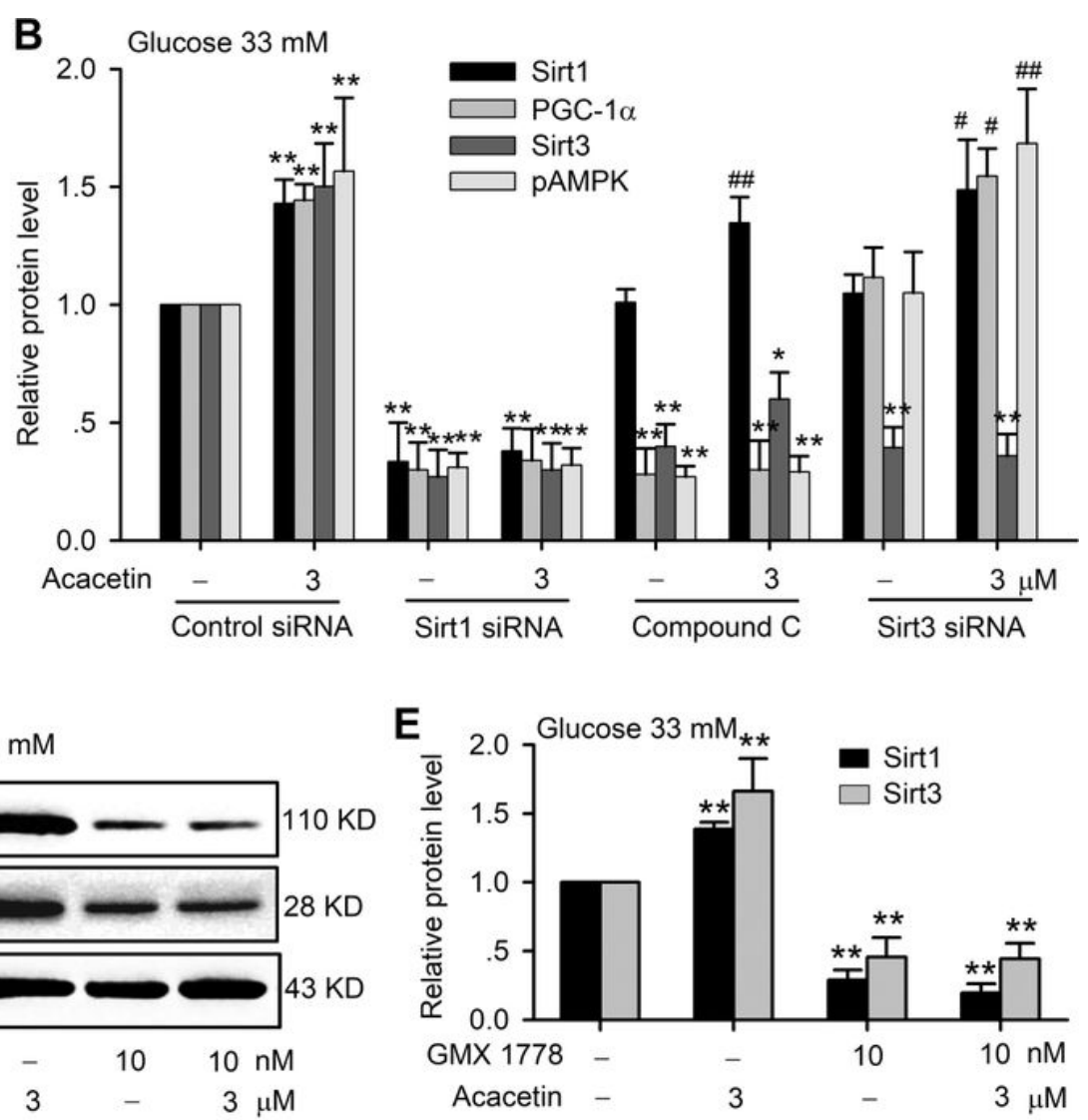

Figure 8 
Sirt1 mediates the vascular protection of acacetin against hyperglycemia-induced injury by activating AMPK/Sirt3/PGC-1a. (A) Western blots of Sirt1, Sirt3, PGC-1a, pAMPK, t-AMPK in HUVECs cultured with $33 \mathrm{mM}$ glucose and transfected with Sirt1 siRNA or Sirt3 siRNA or treated with the AMPK inhibitor Compound $C$ in the absence or presence of $3 \mu \mathrm{M}$ acacetin. (B) Silencing Sirt1, but not Sirt3 or inhibition of pAMPK decreased Sirt1 expression and abolished the Sirt1 activation by acacetin $(n=5$ individual experiments, ${ }^{* *} \mathrm{P}<0.01$ vs. control siRNA $33 \mathrm{mM}$ glucose alone; $\# \mathrm{P}<0.05, \# \# \mathrm{P}<0.01$ vs. $33 \mathrm{mM}$ glucose alone). (C) Acacetin reversal of the high glucose-induced reduction NAD+/NADH ratio was decreased by the NAMPT inhibitor GMX-1778 ( $10 \mathrm{nM})\left(\mathrm{n}=5\right.$ individual experiments, ${ }^{* *} \mathrm{P}<0.01$ vs. $5.5 \mathrm{mM}$ glucose alone; $\# \# P<0.01$ vs. $33 \mathrm{mM}$ glucose alone). (D) Western blots of Sirt1 and Sirt3 in cells cultured with $33 \mathrm{mM}$ glucose medium in the absence or presence of $3 \mu \mathrm{M}$ acacetin or $10 \mathrm{nM}$ GMX-1778 or acacetin plus GMX17788. (E) GMX-1778 decreased the expression of Sirt1 and Sirt3 proteins and abolished the upregulation of Sirt1 and Sirt3 by acacetin ( $n=5, * * P<0.01$ vs. $33 \mathrm{mM}$ glucose alone).

\section{Supplementary Files}

This is a list of supplementary files associated with this preprint. Click to download.

- Supplementalmethods.docx

- Supplementalmethods.docx

- SupplementalFigures12.docx

- SupplementalFigures12.docx 